\title{
Experimental and computational studies on the inhibition performances of benzimidazole and its derivatives for the corrosion of copper in nitric acid
}

\author{
Loutfy H. Madkour ${ }^{1}$ [D $\cdot$ I. H. Elshamy ${ }^{2}$
}

Received: 26 July 2015/ Accepted: 9 November 2015/Published online: 17 February 2016

(c) The Author(s) 2016. This article is published with open access at Springerlink.com

\begin{abstract}
The inhibitive performance of seven synthesized 2-(2-benzimidazolyl)-4 (phenylazo) phenol (BPP_1-7) derivatives was investigated experimentally on the corrosion of copper in $2.0 \mathrm{M} \mathrm{HNO}_{3}$ acid using mass loss, thermometric and DC potentiodynamic polarization techniques. Quantum chemical calculations was investigated to correlate the electronic structure parameters of the investigated benzimidazole derivatives with their inhibition efficiencies (IE\%) values. Global reactivity parameters such as $E_{\mathrm{HOMO}}, E_{\mathrm{LUMO}}$, the energy gap between $E_{\mathrm{LUMO}}$ and $E_{\mathrm{HOMO}}(\Delta E)$, chemical hardness, softness, electronegativity, proton affinity, electrophilicity and nucleophilicity have been calculated and discussed. Molecular dynamics simulation was applied on the compounds, to optimize the equilibrium configurations of the molecules on the copper surface. The areas containing $\mathrm{N}$ atoms are most possible sites for bonding $\mathrm{Cu}(111)$ surface by donating electrons. Binding constant $\left(K_{\mathrm{b}}\right)$, active sites $(1 / y)$, lateral interaction $(f)$, equilibrium constant $\left(K_{\text {ads }}\right)$ and standard free energy of adsorption $\left(\Delta G^{\circ}\right)$ values obtained from either kinetic model and/or Frumkin adsorption isotherm were compared and discussed. Thermodynamic functions and activation parameters such as: $E_{\mathrm{a}}, \Delta H^{*}, \Delta S^{*}$ and $\Delta G^{*}$ at temperatures $303,313,323$ and $333 \mathrm{~K}$ were determined and explained. IE\% values of the examined compounds on $\mathrm{Cu}$ (111) surface followed the order arrangement: $\quad$ BPP_1 $>$ BPP_2 $>$ BPP_3 $>$ BPP_4 >
\end{abstract}

Loutfy H. Madkour

lha.madkour@gmail.com; loutfy_madkour@yahoo.com

1 Chemistry Department, Faculty of Science and Arts, Baljarashi, Al-Baha University, P.O. Box 1988, Al-Baha, Saudi Arabia

2 Chemistry Department, Faculty of Science, Tanta University, Tanta 31527, Egypt
BPP_5 > BPP_6 > BPP_7. The theoretical data obtained as compatible with experimental results showed that the studied benzimidazole derivatives (BPP_1-7) are effective inhibitors for the corrosion of copper in nitric acid solution.

Keywords Corrosion inhibition - Copper .

Benzimidazole derivatives - Quantum chemical calculations - Molecular dynamics simulation - Kinetic . Thermodynamic parameters

\section{Introduction}

Copper and its alloys are widely used materials for their excellent electrical and thermal conductivities in many applications such as electronics, production of wires, sheets, tubes and recently in the manufacture of integrated circuits [1]. Copper is resistant toward the influence of atmosphere and many chemicals; however, it is known that in aggressive media, it is susceptible to corrosion. Copper is relatively noble metal, requiring strong oxidants for its corrosion or dissolution. Nitric acid is one of the most widely used corrosive media that attracted a great deal of research on copper corrosion [2,3]. The use of copper corrosion inhibitors in such conditions is necessary since no protective passive layer can be expected. Experimental techniques are very convenient in the understanding of inhibition mechanism but they are generally expensive and time-consuming. With the improvement of computer hardware and software, density functional theory (DFT) [4, 5] and molecular dynamics (MD) simulation methods in recent times have become fast and powerful tools to predict the corrosion inhibition efficiencies of inhibitor molecules [6-11]. In the conceptual DFT, quantum chemical parameters such as chemical hardness [12, 13], softness [14], 
electronegativity [15], proton affinity [16], electrophilicity [17] and nucleophilicity are considered in the prediction of chemical reactivity or stability. In the calculation of these mentioned chemical properties, Koopmans Theorem [18] provides great facilities to computational and theoretical chemists. According to this theory, ionization energy and electron affinity values of chemical species are associated with their HOMO and LUMO energy values, respectively. Hard-soft acid and base (HSAB) [19] principle introduced by Pearson states that "hard acids prefer to coordinate to hard bases and soft acids prefer to coordinate to soft bases." Polarizable chemical species is defined with soft concept. As can be understood from this classification of Pearson, nitrogen containing structures give electrons easily to metals [20]. In recent years, there is a considerable amount of effort devoted to studying inhibition properties of benzimidazole and its derivatives for metallic corrosion [21]. Benzimidazole is a heterocyclic aromatic organic compound with a bicyclic structure consisting of the fusion of benzene and imidazole rings [10], and hydrogen atoms on the rings can be substituted by other groups or atoms. Some derivatives of benzimidazole have been demonstrated as excellent inhibitors [22-27] for copper and its alloys in acidic solution, and exhibit different inhibition performance with the difference in substituent groups and substituent positions on the imidazole ring [21, 28, 29]. Thus, benzimidazoles are $\mathrm{N}$ - and $\mathrm{O}$-containing compounds and good electron donors; this will enhance their adsorption on the surface of the copper metal and increase binding capabilities. Chemical structures of 2-(2-benzimidazolyl)4(phenylazo) phenol (BPP_1-7) molecules considered in this study are shown in Fig. 1. The corrosion characteristics of copper surface and effectiveness of these compounds as

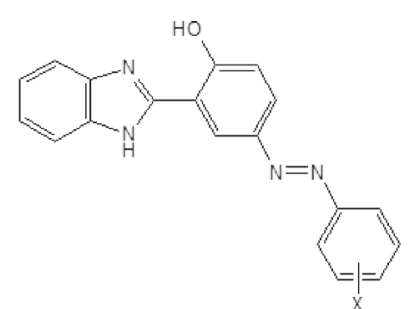

$$
\begin{aligned}
& \mathrm{X}=\mathrm{o}-\mathrm{OCH}_{3} \quad \text { (BPP_1) } \\
& =\mathrm{p}-\mathrm{OCH}_{3} \quad \text { (BPP_2) } \\
& =\mathrm{o}_{-} \mathrm{CH}_{3} \quad \text { (BPP_3) } \\
& =\mathrm{m}-\mathrm{CH}_{3} \quad \text { (BPP_4) } \\
& =\mathrm{m}-\mathrm{OCH}_{3} \quad \text { (BPP_5) } \\
& =\mathrm{p}-\mathrm{NO}_{2} \quad \text { (BPP_6) } \\
& =\mathrm{m}-\mathrm{NO}_{2} \quad \text { (BPP_7) }
\end{aligned}
$$

Fig. 1 Molecular structure of 2-(2-benzimidazolyl)-4 (phenylazo) phenol (BPP_1-7) derivatives chemical inhibitors were described from a quantitative point of view.

\section{Experimental details}

\section{Weight loss measurements}

Weight loss experiments were done according to the standard methods as reported in literature [30].

The corrosion rate, $W$ (expressed in $\mathrm{mg} \mathrm{cm}^{-2} \mathrm{~min}^{-1}$ ) as well as the inhibition efficiency $\left(E_{\mathrm{W}} \%\right)$ over the exposure time period were calculated. The corrosion rates of the copper coupons have been determined for 4-h immersion period at $30 \pm 1{ }^{\circ} \mathrm{C}$ from mass loss, using Eq. (1) where $\Delta m$ is the mass loss, $S$ is the area, and $t$ is the immersion period [31]. The standard deviation of the observed weight loss was $\pm 1 \%$. The percentage protection efficiency $\left(E_{\mathrm{W}} \%\right)$ was calculated according the relationship Eq. (2) where $W$ and $W_{\text {inh }}$ are the corrosion rates of copper without and with the inhibitor, respectively [32]:

$W=\frac{\Delta m}{\text { S.t. }}$

The corrosion rate $W$ (expressed in $\mathrm{mg} \mathrm{cm}^{-2} \mathrm{~min}^{-1}$ ) as well as the inhibition efficiency $\left(E_{\mathrm{W}} \%\right)$ over the exposure time period were calculated according to the following equation:

$E_{\mathrm{W}} \%=\frac{W-W_{\mathrm{inh}}}{W} \times 100$.

\section{Thermometric measurements}

The reaction vessel used was basically the same as that described recently in the previous article [30]. The reaction number $(\mathrm{RN})$ and the reduction in reaction number (\% red $R N$ ) were calculated using the following Eqs. (3) and (4):

$\mathrm{RN}=\frac{\left(T_{\max }-T_{i}\right)}{t}$

$\%$ reduction in $\mathrm{RN}=\frac{\left(\mathrm{RN}_{\text {free }}-\mathrm{RN}_{\mathrm{inh}}\right)}{\mathrm{RN}_{\text {free }}} \times 100$

where $T_{\max }$ and $T_{\mathrm{i}}$ are the maximum and initial temperatures, respectively; $t$ is the immersion time (in minutes) required to reach $T_{\text {max }} ; R N_{\text {free }}$ and $R N_{\text {inh }}$ are the reaction number in the absence and presence of BPP inhibitors, respectively.

\section{Electrochemical measurements}

The copper metal (99.98\% Copper Egyptian Company) was used as foils with a surface area of $2.54 \mathrm{~cm}^{2}$, 
containing $<0.001 \% \mathrm{Ag}, \quad 0.001 \% \mathrm{Sn}$ as impurities. Description details of the electrochemical corrosion cell were explained in my published papers [33, 34].

The corrosion rate $R_{\text {corr }}$ in milli-inches per year (MPY) can be obtained from the following equation:

$R_{\text {corr }}=0.13 I_{\text {corr }} E \cdot W / d$

where $I_{\text {corr }}$ is the corrosion current density $\left(\mu \mathrm{A} \mathrm{cm}{ }^{-2}\right), E \cdot W$ is the equivalent weight of copper and $d$ is its density.

\section{Computational details}

\section{Quantum chemical calculations}

Density functional theory (DFT) is certainly most widely used for the prediction of chemical reactivity of molecules, clusters and solids. It is important to note that DFT methods have become very popular in recent times [30, 35-37]. In the present study, quantum chemical calculations were performed with complete geometry optimizations using Gaussian-09 software package [38]. Geometry optimization were carried out by B3LYP functional at the 6-31G $(d, p)$ basis set [39-42] and at the density functional theory (DFT) level. Frequency calculations were carried at the same levels of the theory to characterize the stationary points as local minima. The molecular orbital structures and energies were also calculated at the B3LYP method with $6-31+\mathrm{G}^{* *}$ basis sets for both HOMO and LUMO levels.

In the conceptual density functional theory, chemical reactivity indices such as chemical hardness, electronegativity, chemical potential are defined as derivatives of the electronic energy (E) with respect to number of electrons (N) at a constant external potential, $v(r)$. Within the framework of this theory, mentioned chemical properties are given as [43, 44]:

$\chi=-\mu=-\left(\frac{\partial E}{\partial N}\right)_{v(r)}$

$\eta=\frac{1}{2}\left(\frac{\partial \mu}{\partial N}\right)_{v(r)}=\frac{1}{2}\left(\frac{\partial^{2} E}{\partial N^{2}}\right)_{v(r)}$

With the help of finite differences method, for chemical hardness, electronegativity and chemical hardness, the following expressions based on first vertical ionization energy and electron affinity values of chemical species are given.

$\eta=\frac{I-A}{2}$

$\chi=-\mu=\frac{I+A}{2}$
According to Koopmans's Theorem [18], the negative of the highest occupied molecular orbital energy and the negative of the lowest unoccupied molecular orbital energy corresponds to ionization energy and electron affinity, respectively $\left(-E_{\mathrm{HOMO}}=I\right.$ and $\left.-E_{\mathrm{LUMO}}=A\right)$. As a result of this theorem, chemical hardness and chemical potential can be expressed as [45]:

$\mu=\frac{E_{\mathrm{LUMO}}+E_{\mathrm{HOMO}}}{2}$

$\eta=\frac{E_{\mathrm{LUMO}}-E_{\mathrm{HOMO}}}{2}$

The global softness is defined as the inverse of the global hardness and this quantity is given as [14]:

$S=\frac{1}{\eta}=-\left(\frac{\partial N}{\partial \mu}\right)_{v(r)}$

The global electrophilicity index $(\omega)$ introduced by Parr [46] is the inverse of nucleophilicity and is given below in Eq. 8. From the light of this index, electrophilic power of a chemical compounds is associated with its electronegativity and chemical hardness. Nucleophilicity $(\varepsilon)$ is the inverse of the electrophilicity as is given below in Eq. (14) [17]:

$\varepsilon=1 / \omega$

$\omega=\frac{\mu^{2}}{2 \eta}=\frac{\chi^{2}}{2 \eta}$.

\section{Molecular dynamics simulations}

Molecular dynamics (MD) simulation is very popular for the investigation regarding the interaction between the inhibitor molecule and the concerned metal surface. The interaction between inhibitors and the copper surface was simulated using Forcite module of Materials Studio 6.0 program developed by Accelrys Inc. [47]. Herein, we had chosen the $\mathrm{Cu}$ (111) surface to simulate the adsorption process. Five layers of copper atoms were used to ensure that the depth of the surface was greater than the non-bond cutoff radius used in the calculation. The MD simulation was performed at $298 \mathrm{~K}$ controlled by the Andersen thermostat, NVT ensemble, with a time step of $1.0 \mathrm{fs}$ and simulation time of $1000 \mathrm{ps}$, using the COMPASS forcefield [48]. Non-bond Interactions, Van der Waals and electrostatic, were set as atom-based summation method and Ewald summation method, respectively, with a cutoff radius of $1.55 \mathrm{~nm}$. Details of simulation process can be referred to some previous literature $[49,50]$.

The interaction energy between the inhibitor molecules and the $\mathrm{Cu}$ (111) surface is calculated by Eq. (15).

$E_{\text {interaction }}=E_{\text {total }}-\left(E_{\text {surface }}+E_{\text {inhibitor }}\right)$ 
Herein, the total energy of the surface and inhibitor molecule is designated as $E_{\text {total }}, E_{\text {surface }}$ is the surface energy without the inhibitor and $E_{\text {inhibitor }}$ is the energy of the adsorbed inhibitor on the surface. The binding energy of the inhibitor molecule is expressed as $E_{\text {binding }}=-E_{\text {interaction }}$.

Obviously, a larger $E_{\text {binding }}$ implies that the corrosion inhibitor combines with the copper surface more easily and tightly, and a higher and spontaneous inhibitive performance of the benzimidazole (BPP_1-7) derivatives.

\section{Results and discussion}

\section{Chemical measurements}

\section{Weight loss measurements}

The value of percentage inhibition efficiency $\left(E_{\mathrm{W}} \%\right)$ and corrosion rate $\left(W_{\text {corr }}\right)$ obtained from weight loss method at different concentrations of (BPP_1) in $2.0 \mathrm{M} \mathrm{HNO}_{3}$ at 303 $\mathrm{K}$ are summarized in Table 1 and shown in Fig. 2. It is evident from Table 1 that the corrosion rate is decreased from $1.87 \mathrm{mg} / \mathrm{cm}^{2} \mathrm{~h}$ to $0.11 \mathrm{mg} / \mathrm{cm}^{2} \mathrm{~h}$ on the addition of $10^{-3} \mathrm{M}$ of BPP_1. The corrosion data for benzimidazole (BPP_1-7) inhibitors are reported in Table 2. According to this data, it is clear that the addition of BPP compounds reduces the corrosion rate of copper in nitric acid solution. The variation of inhibition efficiency with increase in inhibitor concentrations is shown in Fig. 3. It was observed that BPP_1 inhibits the corrosion of copper in $\mathrm{HNO}_{3}$ solution, at all concentrations, i.e., from $10^{-9}$ to $10^{-3} \mathrm{M}$. Maximum inhibition efficiency was shown at $10^{-3} \mathrm{M}$ concentration of the inhibitor in $2.0 \mathrm{M} \mathrm{HNO}_{3}$ to depend on the substituents.

Table 1 Corrosion parameters for copper in aqueous solution of $2.0 \mathrm{M} \mathrm{HNO}_{3}$ in the absence and presence of different concentrations of BPP_1 from weight loss measurements at $303 \mathrm{~K}$ for $4 \mathrm{~h}$

\begin{tabular}{lllll}
\hline Inhibitor & Conc $(\mathrm{M})$ & $W_{\text {corr }}\left(\mathrm{mg} / \mathrm{cm}^{2} \mathrm{~h}\right)$ & $E_{\mathrm{W}}(\%)$ & $\theta$ \\
\hline Blank & $2.0 \mathrm{M} \mathrm{HNO}_{3}$ & 1.87 & - & - \\
BPP_1 & $10^{-9}$ & 1.46 & 22 & 0.22 \\
& $10^{-8}$ & 1.38 & 26 & 0.26 \\
& $10^{-7}$ & 1.33 & 29 & 0.29 \\
& $10^{-6}$ & 1.27 & 32 & 0.32 \\
& $5 \times 10^{-6}$ & 1.14 & 39 & 0.39 \\
& $10^{-5}$ & 1.08 & 42 & 0.42 \\
& $5 \times 10^{-5}$ & 0.67 & 64 & 0.64 \\
& $10^{-4}$ & 0.24 & 87 & 0.87 \\
& $10^{-3}$ & 0.11 & 94 & 0.94 \\
\hline
\end{tabular}

Table 2 Corrosion rate of copper in $2.0 \mathrm{M} \mathrm{HNO}_{3}$ with and without benzimidazole (BPP_1-7) additives at $10^{-4} \mathrm{M}$, and the corresponding inhibition efficiency from weight loss measurements at $303 \mathrm{~K}$ for $4 \mathrm{~h}$

\begin{tabular}{lll}
\hline Solution & $W_{\text {corr }}\left(\mathrm{mg} / \mathrm{cm}^{2} \mathrm{~h}\right)$ & $E_{\mathrm{W}}(\%)$ \\
\hline Blank & 1.87 & - \\
BPP_1 & 0.24 & 87 \\
BPP_2 & 0.52 & 72 \\
BPP_3 & 0.56 & 70 \\
BPP_4 & 0.60 & 68 \\
BPP_5 & 0.77 & 59 \\
BPP_6 & 0.84 & 55 \\
BPP_7 & 0.88 & 53 \\
\hline
\end{tabular}

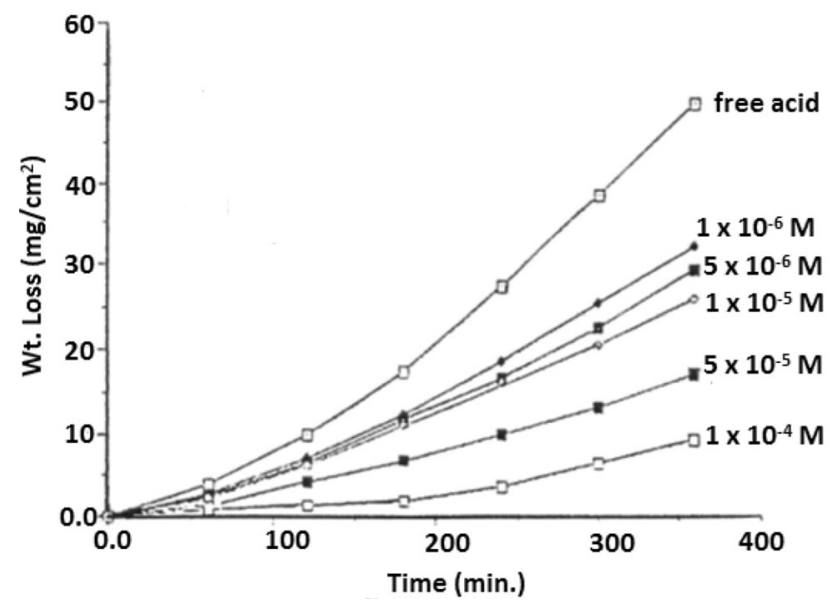

Fig. 2 Variation of weight loss against time for copper corrosion in $2.0 \mathrm{M} \mathrm{HNO}_{3}$ in the presence of different concentrations of BPP_1 at $303 \mathrm{~K}$

\section{Thermometric measurements}

In this method, the temperature change of the system involving copper dissolution in $2.0 \mathrm{M} \mathrm{HNO}_{3}$ was followed without and with different concentrations of the investigated BPP_1 derivative, as tabulated in Table 3 and shown in Fig. 4 for BPP_1 as an example for the additives. An incubation period is first recognized along which the temperature rises gradually with time. The temperature-time curves provide a mean of differentiating between weak and strong adsorption. The thermometric data for all benzimidazole (BPP_1-7) derivatives are depicted in Table 4. It is evident that the dissolution of copper in $2.0 \mathrm{M} \mathrm{HNO}_{3}$ starts from the moment of immersion. On increasing the concentration of the inhibitor from $10^{-6}$ to $10^{-4} \mathrm{M}$ the value of $T_{\max }$ decreases, whereas the time $(t)$ required for reaching $T_{\max }$ increases, and both factors cause a large 
Fig. 3 Variation of inhibition efficiency $E_{\mathrm{W}}(\%)$ on copper with different concentrations of BPP_1 inhibitor in $2.0 \mathrm{M} \mathrm{HNO}_{3}$ at $303 \mathrm{~K}$ for $4 \mathrm{~h}$ immersion time

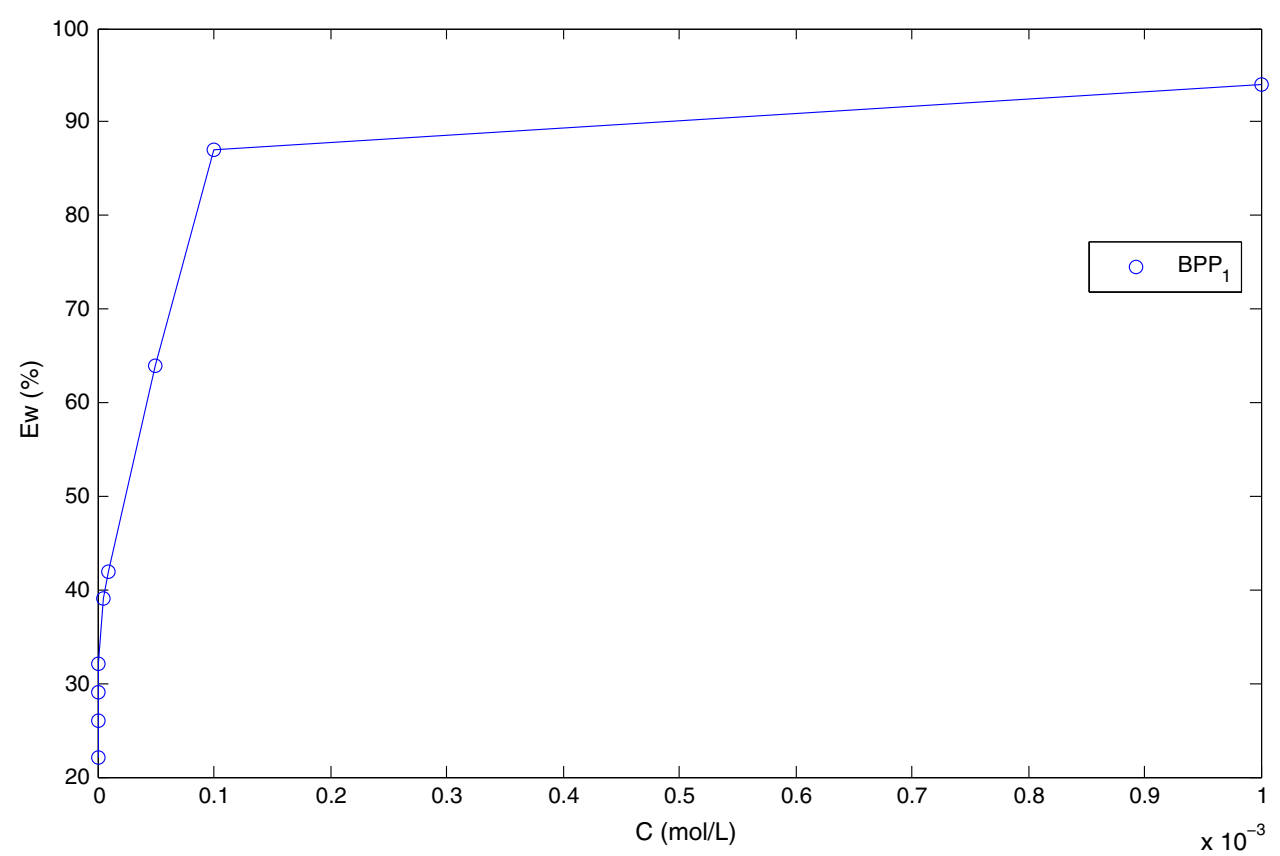

Table 3 Effect of different concentrations of BPP_1 on the thermometric parameters of copper in $2.0 \mathrm{M} \mathrm{HNO}_{3}$

\begin{tabular}{llllllllll}
\hline Conc. $(\mathrm{M})$ & $\log C$ & $T_{\mathrm{i}}\left({ }^{\circ} \mathrm{C}\right)$ & $T_{\max }\left({ }^{\circ} \mathrm{C}\right)$ & $t(\min )$ & $\Delta t(\min )$ & $\log \Delta t$ & $\theta$ & R.N. & $\%$ red in R.N. \\
\hline $2.0 \mathrm{M} \mathrm{HNO}_{3}$ & & 26.0 & 35.5 & 120 & & & & 0.079 & \\
$10^{-6}$ & -6.0 & 25.9 & 34.6 & 165 & 45 & 1.65 & 0.342 & 0.052 & 34.17 \\
$5 \times 10^{-6}$ & -5.3 & 26.0 & 33.5 & 180 & 60 & 1.77 & 0.481 & 0.041 & 48.10 \\
$10^{-5}$ & -5.0 & 26.0 & 32.1 & 210 & 90 & 1.95 & 0.658 & 0.027 & 65.82 \\
$5 \times 10^{-5}$ & -4.3 & 26.1 & 31.4 & 225 & 105 & 2.02 & 0.708 & 0.023 & 70.88 \\
$10^{-4}$ & -4.0 & 26.2 & 29.6 & 240 & 120 & 2.07 & 0.823 & 0.014 & 82.27 \\
\hline
\end{tabular}

decrease in $R N$ and increasing of \% red $R N$ of the system, as shown in Tables 3 and 4. This indicates that the synthesized BPP_1-7 additives retard the dissolution presumably by strong adsorption onto the copper surface. The extent of inhibition depends on the degree of the surface coverage $(\theta)$ of the copper surface with the adsorbate. Strehblow and Titze [51] showed that the $\mathrm{Cu}$ passive layer consists of a duplex structure of oxides, with an inner cuprous oxide and an outer cupric hydroxide. Copper will not corrode in non-oxidizing acidic environments, since hydrogen evolution is not a part of its corrosion process. However, when oxygen or other oxidants such as $\mathrm{Fe}^{3+}$ and $\mathrm{NO}_{3}{ }^{-}$ions are present, corrosion becomes important. Copper oxides are stable only in the $\mathrm{pH}$ range of 8-12 [52], but not in acidic solutions [Eqs. (16) and (17)] in which surface roughening can occur:

$\mathrm{Cu}_{2} \mathrm{O}+2 \mathrm{H}^{+} \rightleftarrows 2 \mathrm{Cu}^{+}+\mathrm{H}_{2} \mathrm{O}$

$\mathrm{CuO}+2 \mathrm{H}^{+} \rightleftarrows \mathrm{Cu}^{2+}+\mathrm{H}_{2} \mathrm{O}$

Moreover, $\mathrm{Cu}^{+}$ions can undergo disproportionation according to Eq. (18).
$2 \mathrm{Cu}^{+} \rightleftarrows \mathrm{Cu}^{2+}+\mathrm{Cu}$

These reactions take place along the incubation period. The heat evolved from the above reactions accelerates further dissolution of the oxide and activates the dissolution of the copper metal exposed to the aggressive medium. The thermometric parameters measured experimentally such as $R N$, time delay $(\Delta t)$ and/or log $(\Delta t)$ versus molar concentration of the investigated benzimidazole (BPP_1-7) derivatives confirms and support a two-step adsorption process, at first a monolayer of the adsorbed is formed on the copper electrode surface, and then it is followed by the adsorption of a second adsorbed layer or a chemical reaction leading to the deposition of the BPP-Cu complex on the metal surface. The plot of $\Delta t$ and/or $\log (\Delta t)$ as a function of $\log C_{\text {In }}$ yields a linear relation shape for the first region of the curve then a region of constancy; this reveals the completion of the adsorbed monolayer of the inhibitor. In thermometric measurements \% red $R N$ values are taken as the measure for the corrosion inhibition efficiency (IE\%). Plots of \% red RN versus molar concentration $\left(C_{\mathrm{In}}\right)$ of the additives for copper corrosion in $2.0 \mathrm{M} \mathrm{HNO}_{3}$ are invariably sigmoidal in nature 


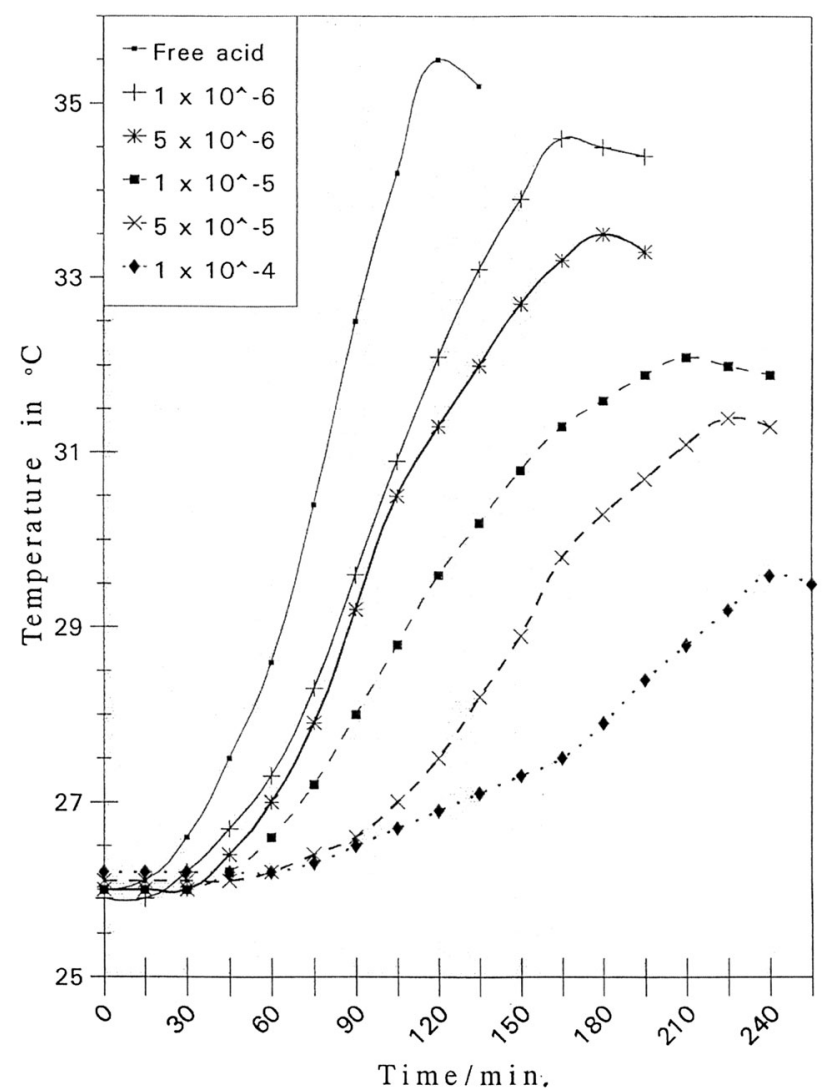

Fig. 4 Temperature versus time curves of copper corrosion in $2.0 \mathrm{M}$ $\mathrm{HNO}_{3}$ solution in the absence and presence of different concentrations of BPP_1

as shown in Fig. 5. The inhibition efficiency of the benzimidazole (BPP_1-7) derivatives depends on many factors, including the molecular size, heat of hydrogenation, mode of interaction with copper electrode surface, formation of metallic complexes and the charge density on the adsorption sites. Adsorption is expected to take place primarily through functional groups; essentially $\mathrm{OH}$ and $\mathrm{OCH}_{3}$ would depend on its charge density. The thermometric technique cannot be applied for the copper corrosion in alkaline media because of the formation of $\mathrm{Cu}_{2} \mathrm{O}, \mathrm{CuO}$ and $\mathrm{Cu}(\mathrm{OH})_{2}$ oxide films on the copper electrode surface, which formed only in near neutral and slightly alkaline solutions $(\mathrm{pH}=8-12)$ [52].

\section{Electrochemical measurements}

\section{Potentiodynamic polarization measurement}

Potentiodynamic measurements were performed in the presence of different concentrations of 2-(2-benzimidazolyl)-4(phenylazo) phenol (BPP_1-7) derivatives. The results are tabulated in Tables 5, 6 and plotted in Figs. 6, 7, which show that the anodic and cathodic polarization curves of copper in $2.0 \mathrm{M} \mathrm{HNO}_{3}$ solution, without and with BPP_1 derivative at $303 \mathrm{~K}$. It is observed that both the cathodic curves and anodic curves show lower current density in the presence of the investigated inhibitors (BPP) than that of the blank. This indicates that the benzimidazoles inhibit the corrosion rate. Potentiokinetic polarization curves were plotted for copper corrosion in $2.0 \mathrm{M} \mathrm{HNO}_{3}$ in the presence of the benzimidazole (BPP_1-7) derivatives as given in Fig. 7. A linear region with apparent Tafel was observed. The cathodic reaction was activation controlled and the addition of the compounds tested decreased the current densities in large anodic and cathodic domains of potential. The results indicated that the BPP_1-7 derivatives acted as mixed-type inhibitors. Generally, the addition of mixed inhibitors in solution does not change corrosion potential significantly because they inhibit both the anodic and cathodic reactions. Small changes in potentials can be a result of the competition of the anodic and the cathodic inhibiting reactions, and of the copper surface condition. The results of Fig. 7 illustrate that all BPP_1-7 derivatives bring down the corrosion current without causing any considerable change in the corrosion mechanism. According to corrosion theory, the rightward shift of the cathodic curves reveal that corrosion is mainly accelerated by cathode reactions. $\mathrm{HNO}_{3}$ is a strong copper oxidizer capable of rapidly attacking copper. The potentiodynamic behavior of pure $\mathrm{Cu}$ in near neutral and slightly alkaline solutions exhibits three anodic peaks associated with the formation of $\mathrm{Cu}_{2} \mathrm{O}, \mathrm{CuO}$ and $\mathrm{Cu}(\mathrm{OH})_{2} \cdot \mathrm{Cu}_{2} \mathrm{O}$ is first formed [Eq. (19)], which subsequently oxidizes to $\mathrm{CuO}$ [Eq. (20)] or, at more positive potentials, to $\mathrm{Cu}(\mathrm{OH})$ 2 [Eq. (21)].
Table 4 Effect of different concentrations of benzimidazole (BPP_1-7) derivatives on the inhibition efficiency IE\% of copper in $2.0 \mathrm{M} \mathrm{HNO}_{3}$ solution as determined by thermometric technique

\begin{tabular}{llllllll}
\hline Conc. (M) & \multicolumn{7}{l}{ \% red in R.N. } \\
\cline { 2 - 7 } & BPP_1 & BPP_2 & BPP_3 & BPP_4 & BPP_5 & BPP_6 & BPP_7 \\
\hline $1 \times 10^{-6}$ & 34.17 & 32.91 & 30.37 & 35.44 & 13.92 & 34.17 & 15.18 \\
$5 \times 10^{-6}$ & 48.10 & 41.77 & 43.03 & 44.30 & 26.58 & 45.56 & 29.11 \\
$1 \times 10^{-5}$ & 65.82 & 63.29 & 48.10 & 51.89 & 37.97 & 49.36 & 39.24 \\
$5 \times 10^{-5}$ & 70.88 & 67.08 & 54.49 & 56.96 & 49.36 & 53.16 & 46.83 \\
$1 \times 10^{-4}$ & 82.27 & 74.68 & 68.30 & 63.29 & 62.02 & 58.22 & 55.65 \\
\hline
\end{tabular}


Fig. 5 Effect of concentration of BPP_1 on \% reduction in $\mathrm{RN}$ for copper in $2.0 \mathrm{M} \mathrm{HNO}_{3}$

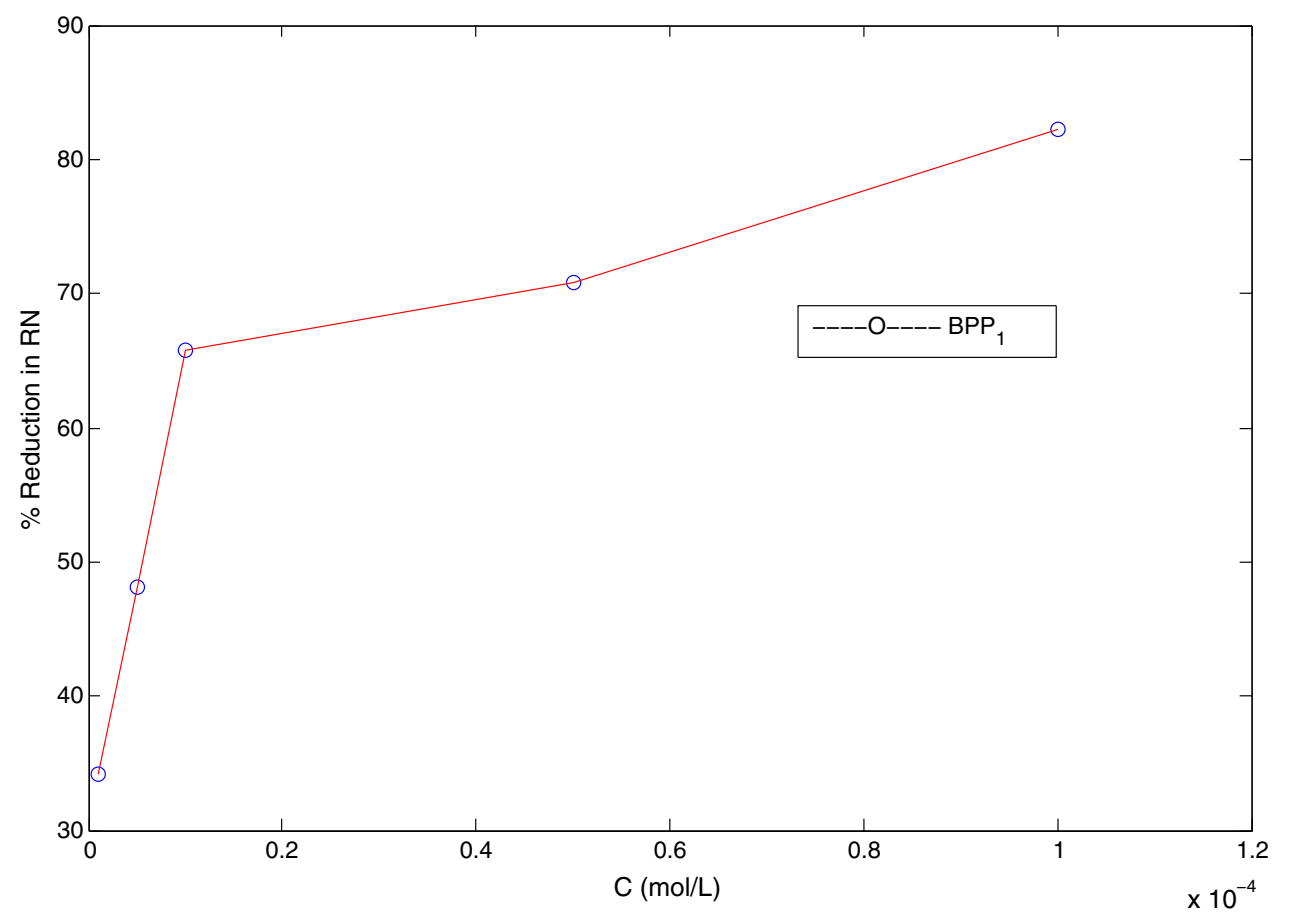

Table 5 Polarization corrosion parameters of $\mathrm{Cu}$ in $2.0 \mathrm{M} \mathrm{HNO}_{3}$ solution containing BPP_1 at $295 \mathrm{~K}$

\begin{tabular}{|c|c|c|c|c|c|c|c|c|c|}
\hline Inhibitor type & $\begin{array}{l}\text { Conc. } \\
\text { (M) }\end{array}$ & $\begin{array}{l}R_{\mathrm{p}} \\
\left(\Omega \mathrm{cm}^{2}\right)\end{array}$ & $\begin{array}{l}I_{\text {corr }}(\mu \mathrm{A} \\
\left.\mathrm{cm}^{-2}\right)\end{array}$ & $\begin{array}{l}R_{\text {corr }} \\
\text { (MPY) }\end{array}$ & $\begin{array}{l}-E_{\text {corr }} \\
(\mathrm{mV}(\mathrm{SCE}))\end{array}$ & $\begin{array}{l}\beta_{\mathrm{c}} \\
\left(\mathrm{V} \operatorname{dec}^{-1}\right)\end{array}$ & $\begin{array}{l}\beta_{\mathrm{a}} \\
\left(\mathrm{V} \operatorname{dec}^{-1}\right)\end{array}$ & $\begin{array}{l}E_{R_{\mathrm{p}}} \% \\
\left(\Omega \mathrm{cm}^{2}\right)\end{array}$ & IE\% \\
\hline $2.0 \mathrm{M} \mathrm{HNO}_{3}$ & - & 7.053 & 7236 & 6671 & 61 & 0.233 & 0.237 & - & - \\
\hline \multirow{3}{*}{$\begin{array}{l}2.0 \mathrm{M} \\
\quad \mathrm{HNO}_{3}+(\text { BPP_1) }\end{array}$} & $10^{-7}$ & 13.52 & 3468 & 3197 & 68 & 0.196 & 0.241 & 47.83 & 52.07 \\
\hline & $10^{-6}$ & 17.74 & 2317 & 2137 & 47 & 0.176 & 0.204 & 60.24 & 67.96 \\
\hline & $5 \times 10^{-6}$ & 221.7 & 104.8 & 96.63 & 10 & 0.160 & 0.081 & 96.82 & 98.55 \\
\hline
\end{tabular}

Table 6 Polarization corrosion parameters of $\mathrm{Cu}$ in $2.0 \mathrm{M} \mathrm{HNO}_{3}$ solution containing $1 \times 10^{-6} \mathrm{M}$ of benzimidazole (BPP_1-7) derivatives at $303 \mathrm{~K}$

\begin{tabular}{|c|c|c|c|c|c|c|c|c|}
\hline Inhibitor type & $R_{\mathrm{p}}\left(\Omega \mathrm{cm}^{2}\right)$ & $I_{\text {corr }}\left(\mu \mathrm{A} \mathrm{cm}^{-2}\right)$ & $R_{\text {corr }}(\mathrm{MPY})$ & $E_{\text {corr }}(\mathrm{mV}(\mathrm{SCE}))$ & $\beta_{\mathrm{c}}\left(\mathrm{V} \mathrm{dec}{ }^{-1}\right)$ & $\beta_{\mathrm{a}}\left(\mathrm{V} \mathrm{dec}{ }^{-1}\right)$ & $E_{R_{\mathrm{p}}} \%\left(\Omega \mathrm{cm}^{2}\right)$ & IE\% \\
\hline $2.0 \mathrm{M} \mathrm{HNO}_{3}$ & 6.360 & 7287 & 6719 & 29 & 0.302 & 0.165 & - & - \\
\hline BPP_1 & 45.08 & 235 & 731.3 & 23 & 0.177 & 0.154 & 85.9 & 96.8 \\
\hline BPP_2 & 75.34 & 406.7 & 374.9 & 12 & 0.162 & 0.125 & 91.5 & 94.4 \\
\hline BPP_3 & 63.36 & 507 & 467.4 & 7 & 0.145 & 0.151 & 89.9 & 93.0 \\
\hline BPP_4 & 30.93 & 1180 & 1206 & 26 & 0.185 & 0.188 & 79.4 & 83.8 \\
\hline BPP_5 & 76.40 & 397.2 & 366.2 & 7 & 0.202 & 0.107 & 91.7 & 94.5 \\
\hline BPP_6 & 147.9 & 320 & 295.1 & 6 & 0.204 & 0.234 & 95.7 & 95.6 \\
\hline BPP_7 & 113.0 & 242.5 & 290 & -3 & 0.124 & 0.129 & 94.4 & 96.6 \\
\hline
\end{tabular}

$$
\begin{aligned}
& 2 \mathrm{Cu}+\mathrm{H}_{2} \mathrm{O} \rightleftarrows \mathrm{Cu}_{2} \mathrm{O}+2 \mathrm{H}^{+}+2 \mathrm{e}^{-} \\
& \mathrm{Cu}_{2} \mathrm{O}+\mathrm{H}_{2} \mathrm{O} \rightleftarrows 2 \mathrm{CuO}+2 \mathrm{H}^{+}+2 \mathrm{e}^{-} \\
& \mathrm{Cu}_{2} \mathrm{O}+3 \mathrm{H}_{2} \mathrm{O} \rightleftarrows 2 \mathrm{Cu}(\mathrm{OH})_{2}+2 \mathrm{H}^{+}+2 \mathrm{e}^{-}
\end{aligned}
$$

Additionally, the potentiodynamic polarization curves in Figs. 6, 7 exhibit no steep slope in the anodic range, meaning that no passive films are formed on the copper surface. Consequently, copper may directly dissolve in 


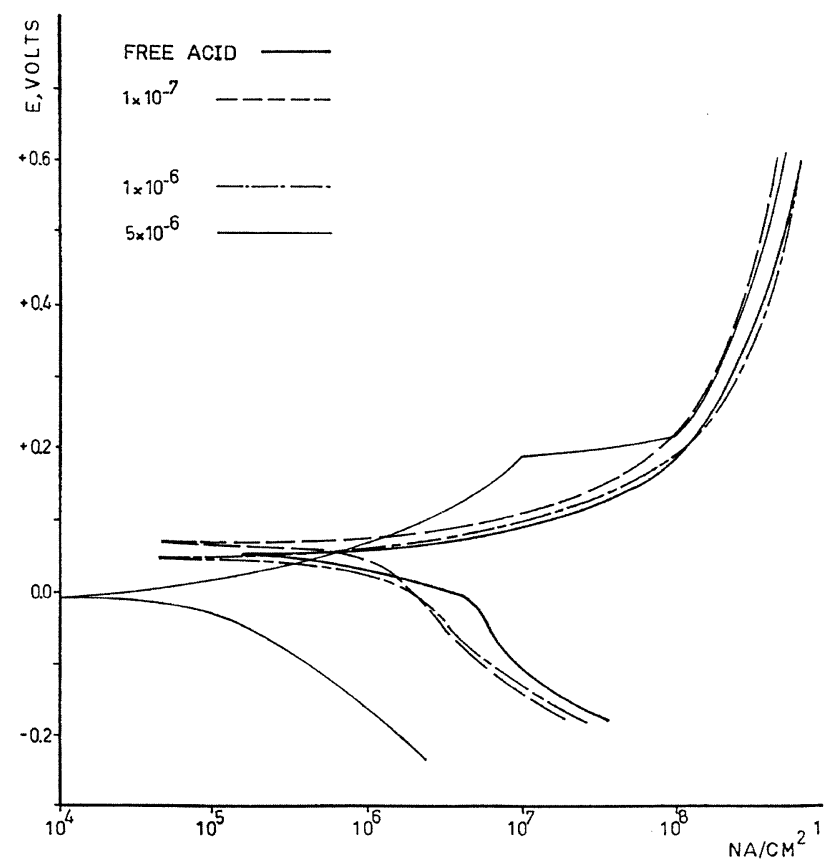

Fig. 6 Cathodic and anodic polarization curves of copper in $2.0 \mathrm{M}$ $\mathrm{HNO}_{3}$ solution with the BPP_1 in different concentrations at $303 \mathrm{~K}$

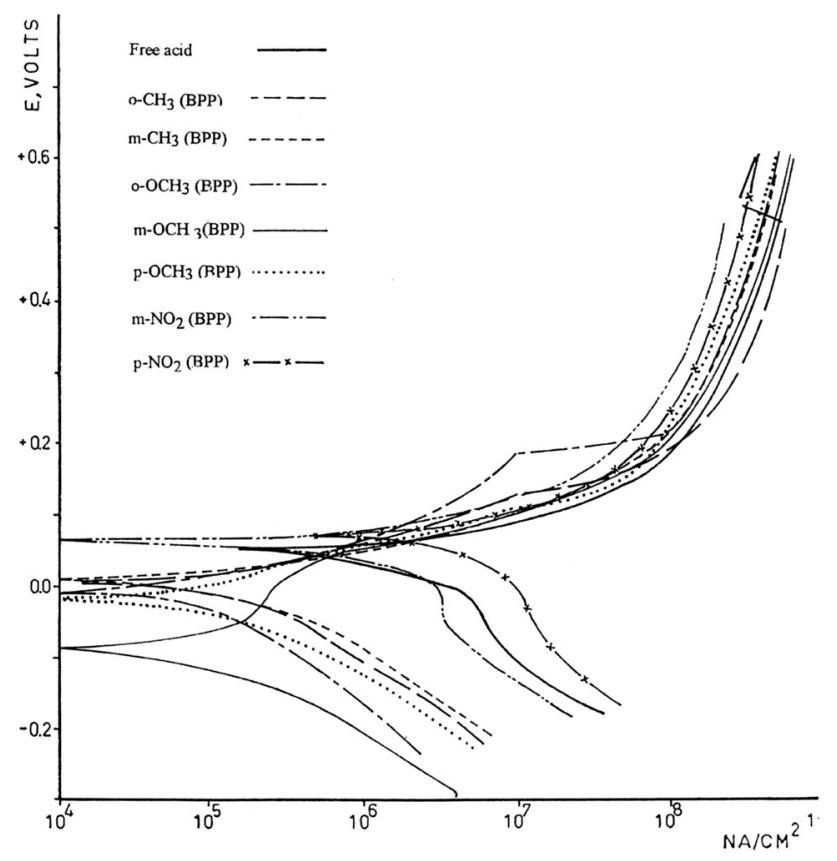

Fig. 7 Potentiodynamic polarization curves of copper in $2.0 \mathrm{M}$ $\mathrm{HNO}_{3}$ solution with the $5 \times 10^{-6} \mathrm{M}$ of different BPP_1-7 inhibitors at $303 \mathrm{~K}$

2.0 $\mathrm{M} \mathrm{HNO}_{3}$ solutions. The Pourbaix diagram for copperwater system is shown in Fig. 8 [53]. It indicates that copper is corroded to $\mathrm{Cu}^{2+}$ in $\mathrm{HNO}_{3}$ solutions, and no oxide film is formed to protect the surface from corrosion. Copper dissolution is, thus, expected to be the dominant

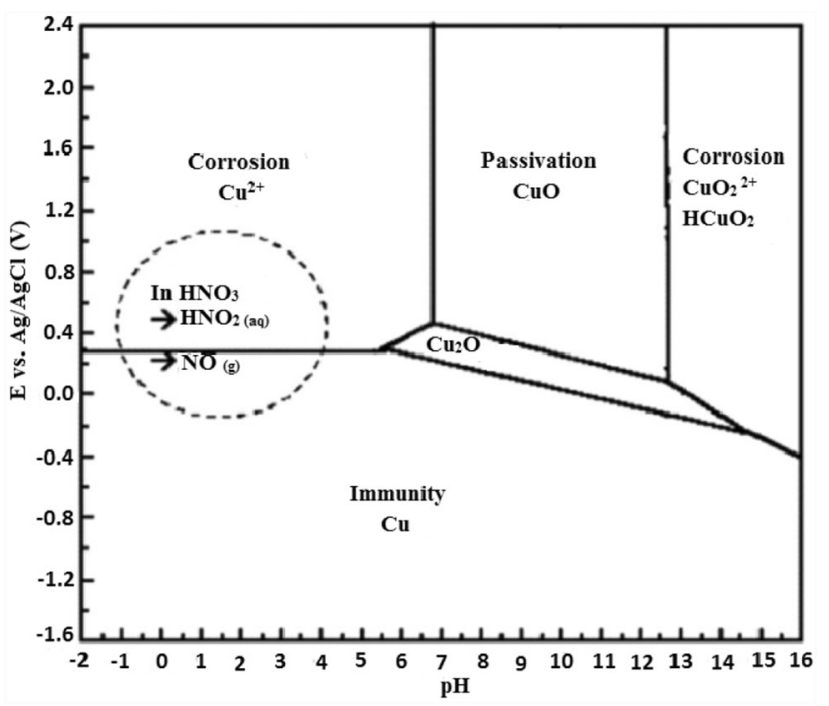

Fig. 8 Potential-pH equilibrium diagram for the system, copperwater, at $25 \pm 1{ }^{\circ} \mathrm{C}$ [53]. The dashed regions and the equilibrium components corresponding to the Pourbaix diagram in $\mathrm{HNO}_{3}$

reaction in $\mathrm{HNO}_{3}$ solutions. The electrochemical reactions for copper in $\mathrm{HNO}_{3}$ solution can be described as follows:

Anodic reaction:

$$
\mathrm{Cu} \rightarrow \mathrm{Cu}^{2+}+2 \mathrm{e}^{-}
$$

Cathodic reactions [54]:

$$
\begin{aligned}
& \mathrm{NO}_{3}^{-}+3 \mathrm{H}^{+}+2 \mathrm{e}^{-} \rightarrow \mathrm{HNO}_{2}+\mathrm{H}_{2} \mathrm{O} \\
& \mathrm{NO}_{3}^{-}+4 \mathrm{H}^{+}+3 \mathrm{e}^{-} \rightarrow \mathrm{NO}+2 \mathrm{H}_{2} \mathrm{O} \\
& \mathrm{O}_{2}+4 \mathrm{H}^{+}+4 \mathrm{e}^{-} \rightarrow 2 \mathrm{H}_{2} \mathrm{O}
\end{aligned}
$$

Figure 7 shows the anodic and cathodic Tafel plots of copper in the presence of all seven studied BPP_1-7 inhibitors, for comparison polarization curves measured in $2.0 \mathrm{M} \mathrm{HNO}_{3}$ acid solution are plotted. These compounds induce an increase in both the cathodic and anodic over voltages and cause a parallel displacement of both the cathodic and the anodic Tafel curves. The results indicate that the presence of the BPP compounds in the solution inhibits both the hydrogen evolution and the anodic dissolution processes. The data suggest that all tested BPP act as mixed-type inhibitors. They also indicate that the BPP molecules are mainly adsorbed at the copper surface via the oxygen and nitrogen centers of adsorption. The corrosion current density, $I_{\text {corr }}$, for copper in free $2.0 \mathrm{M} \mathrm{HNO}_{3}$ acid is $7236\left(\mu \mathrm{A} \mathrm{cm}^{-2}\right)$. Addition of the BPP derivatives tested induces a decrease in both $I_{\text {corr }}$ and $R_{\text {corr }}$ whereas, the $R_{\mathrm{p}}$ values at the same time increases; thus BPP derivatives act as inhibitors for copper dissolution in nitric acid. Table 5 gives the effect of BPP_1 concentrations on corrosion 
parameters. In general, the inhibition efficiency depends on the concentration of the inhibitors. When an external emf was applied, both the cathode and the anode were polarized due to the presence of the nitrogen atom which is responsible for the cathodic behavior and the oxygen atom which is responsible for the anodic behavior. Similar behavior was observed in the presence of different BPP additives, from Table 5, the slopes of cathodic and anodic Tafel lines $\left(\beta_{\mathrm{c}}\right.$ and $\left.\beta_{\mathrm{a}}\right)$ were more changed on increasing the concentration of the tested inhibitor and have the values $\beta_{\mathrm{c}}=0.233-0.160 \mathrm{~V} /$ decade and $\beta_{\mathrm{a}}=0.237-0.081 \mathrm{~V} /$ decade. This behavior indicates that the adsorbed BPP derivatives mechanically screen the coated part of the copper electrode and therefore, protect it from the action of the corrosive medium. BPP inhibitor molecules have the effect on the mechanism of dissolution of copper and cause activation of a part of the surface respect to the corrosive medium. The high Tafel constant values support the probability of the presence of a complicated surface processes involving $\mathrm{Cu}$ ions and BPP molecules. Moreover, Tafel slopes of about $75 \mathrm{mV}$ decade $^{-1}$ can be attributed to a surface kinetic process rather than a diffusion controlled process. Inspection of polarization curves in Figs. 6, 7 show that, it was not possible to evaluate the cathodic Tafel slope as there is no visible linear region that prevents linear extrapolation to $E_{\text {corr }}$ of the cathodic polarization curves. It has been shown that in the Tafel extrapolation method, use of both the anodic and cathodic Tafel regions is undoubtedly preferred over the use of only one Tafel region [55]. However, the corrosion rate can also be determined by Tafel extrapolation of either the cathodic or anodic polarization curve alone. If only one polarization curve alone is used, it is generally the cathodic curve which usually produces a longer and better defined Tafel region. Anodic polarization may sometimes produce concentration effects, due to passivation and dissolution, as noted above, as well as roughening of the surface which can lead to deviations from Tafel behavior. The situation is quite different here; the anodic dissolution of copper in aerated 2.0 $\mathrm{M} \mathrm{HNO}_{3}$ solutions obeys Tafel's law. The anodic curve is, therefore, preferred over the cathodic one for the Tafel extrapolation method. However, the cathodic polarization curve displays a limiting diffusion current due to the reduction of dissolved oxygen. Thus, the cathodic process is controlled, as will be seen latter, by concentration polarization rather than activation polarization, which prevented linear extrapolation of the cathodic curves. The corrosion current densities were obtained here by extrapolation of the anodic polarization curves to $E_{\text {corr }}$. Similar results were reported previously [54, 56, 57]. This irregularity was confirmed by other researchers and can be explained as the superposition of at least two cathodic current contributions: one arises from oxygen reduction and the second one consequential of copper ion re-deposition [58]. It is common practice and it was possible in this case to evaluate $I_{\text {corr }}$ by extrapolation of the anodic polarization curves only to $E_{\text {corr }}$. At more cathodic potential with respect to $E_{\text {corr }}$, the characteristic horizontal line resulting from limiting current density for oxygen reduction can be observed. Because of uncertainty and source of error in the numerical values of the cathodic Tafel slope $\left(\beta_{\mathrm{c}}\right)$ calculated by the software; We did not introduce here $\beta_{\mathrm{c}}$ values recorded by the software. Table 5 gives the values of the associated electrochemical parameters of corrosion such as corrosion current density $I_{\text {corr }}$ which calculated with the extrapolation of the linear parts of Tafel lines, corrosion potential $E_{\text {corr }}$, anodic Tafel constants $\beta_{\mathrm{a}}$. The inhibition efficiency (IE\%) was determined from the polarization curves using the Eq. (26):

$\mathrm{IE} \%=\frac{I_{\text {corr }}-I_{\text {corr }}^{\prime}}{I_{\text {corr }}} \times 100$

$I_{\text {corr }}$ and $I_{\text {corr }}^{\prime}$ are the uninhibited and inhibited corrosion current densities, respectively, determined by extrapolation of the cathodic Tafel lines to corrosion potential $\left(E_{\text {corr }}\right)$. It was clearly seen that cathodic slope was found equal indicating that the reduction of hydrogen did not modified in the presence of the azoles (BPP_1-7) inhibitors. Thus, the presence of the inhibitors at $10^{-6} \mathrm{M}$ leads to decrease in the values of $I_{\text {corr }}$, which was particularly significant in the case of BPP_1 as shown in Table 5. The inhibition properties of BPP derivatives were evaluated also by polarization resistance method. Tables 5 and 6 gathered the corresponding values of polarization resistance $R_{\mathrm{p}}$ and the inhibition efficiency $\left(E_{R_{\mathrm{p}}} \%\right.$ ) which is calculated using the Eq. (27).

$E_{R_{p}} \%=\frac{R_{p}^{\prime}-R_{p}}{R_{p}^{\prime}} \times 100$

$R_{\mathrm{p}}$ and $R_{p}^{\prime}$ are the polarization resistance values without and with inhibitor, respectively. From polarization resistance measurements, the weakest value of $R_{\mathrm{p}}$ is found for copper in 2.0 $\mathrm{M} \mathrm{HNO}_{3}$ without inhibitor. However, the addition of the BPP derivatives in solution leads to an increase in the polarization resistance values. In fact, the value of $R_{\mathrm{p}}$ is $45.08 \Omega \mathrm{cm}^{2}$ in the case of BPP_1 and increases to $\left(63.36 \Omega \mathrm{cm}^{2}\right)$ for BPP_3, then, increases to $\left(76.40 \Omega \mathrm{cm}^{2}\right)$ for BPP_5 and reaches to $\left(147.9 \Omega \mathrm{cm}^{2}\right)$ for BPP_7 as given in Table 6 . This in turn leads to a decrease in corrosion current density $I_{\text {corr }}$ values because this later is inversely proportional to $R_{\mathrm{p}}$. Table 5 show that the increasing of the concentration from $10^{-7}$ to $5 \times 10^{-6} \mathrm{M}$ (BPP_1) reduces significantly the dissolution rate of copper and consequently increasing IE\%. Anodic Tafel constants change with inhibitor concentration, which is an indication of its effect on the copper dissolution reaction. No definite 
trend was observed in the shift of $E_{\text {corr }}$ values, in the presence of various concentrations of BPP derivatives in 2.0 $\mathrm{M} \mathrm{HNO}_{3}$ solutions. BPP_1-7 derivatives may be classified as mixed-type inhibitors. Figure 7 shows that the polarization curves of copper in the presence of $5 \times 10^{-6}$ $\mathrm{M}$ of all BPP_ 1-7 inhibitors. The arrangement of IE\% follows the order: BPP_1 $>$ BPP_2 $>$ BPP_3 $>$ BPP_4 $>$ BPP_5 $>$ BPP_6 $>$ BPP_7. In the case of p-nitro derivative BPP_6, the inhibition efficiency (IE\%) firstly increases, then sharply decreases by increasing the inhibitor concentration. This can be explained on the basis that, the $\mathrm{p}-\mathrm{NO}_{2}$ group characterized by critical inhibitor concentration $(C I C)$ as well as the critical micelle concentration $(C M C)$. The inhibition efficiency (IE\%) of BPP derivatives depend on many factors, including the number of adsorption sites, their charge density, molecular size and mode of interaction with the copper metal surface. Retardation of copper dissolution by the BPP substituents is expected to be due to their adsorption on the copper surface via oxygen and nitrogen atoms. In general $\mathrm{o}-\mathrm{OCH}_{3}$ 2-(2(benzimidazolyl)-4-(phenylazo)-phenol (BPP_1) inhibitor is found to be the most efficient inhibitor; this may be due to the position of $-\mathrm{OCH}_{3}$ group at ortho position in the phenylazo and its nature to the nearest on the phenol ring. This aids the more flat of the molecules and coverage the copper substrate to be more possible. This flat formation and coating effect decreases in the case of $\mathrm{m}-\mathrm{OCH}_{3}$ derivative.

\section{Adsorption isotherm and thermodynamic activation parameters}

The adsorption isotherm can be determined by assuming that inhibition effect is due mainly to the adsorption at metal/solution interface. Basic information on the adsorption of inhibitors on the metal surface can be provided by adsorption isotherm. In order to obtain the isotherm, the fractional surface coverage values $(\theta)$ as a function of inhibitor concentration must be obtained. The values of $\theta$ can be easily determined from the weight loss measurements by the ratio $E_{\mathrm{W}} \% / 100$, where $E_{\mathrm{W}} \%$ is inhibition efficiency obtained by weight loss method. So it is necessary to determine empirically which isotherm fits best to the adsorption of inhibitors on the copper surface. Several adsorption isotherms (viz., Frumkin, Langmuir, Temkin, and Freundlich) were tested. Data were tested graphically by fitting to various isotherms. Figure 9 shows the dependence of the fraction $(\theta)$ as function of the logarithm of the concentration of BPP_1. The obtained plot is consistent with an S-shape adsorbed isotherm for BPP_1 showing an adsorption on the copper surface according to the Frumkin isotherm. $\frac{\theta}{1-\theta} \exp (-f \theta)=K_{\mathrm{ads}} C$

with

$$
\begin{gathered}
K_{\mathrm{ads}}=\frac{1}{55.5} \exp \left(\frac{-\Delta G_{\mathrm{ads}}^{\circ}}{\mathrm{RT}}\right) \text { or } \\
\Delta G_{\mathrm{ads}}^{\circ}=-\mathrm{RT}\left(\ln 55.5 K_{\mathrm{ads}}\right)
\end{gathered}
$$

where $C$ is the concentration of the adsorbed substance in the bulk of the solution and $\theta$ is the degree of surface coverage of the metal surface by the inhibitor. $K_{\text {ads }}$ is the modified equilibrium constant of the adsorption process, $f$ is a constant depending on intermolecular interactions in the adsorption layer and on the heterogeneity of the surface, $R$ is the universal gas constant and $T$ is the absolute temperature. The value 55.55 in the above equation is the concentration of water in solution in mol L ${ }^{-1}$. The values of $K_{\text {ads }}$ and $\Delta G_{\text {ads }}^{\circ}$ were calculated at $303 \mathrm{~K}$ and are listed in Table $7 . \Delta G_{\text {ads }}^{\circ}$ is the free energy of adsorption and $f$ is a function of adsorption energy. The average value of $K, f$ and $\Delta G_{\text {ads }}^{\circ}$ calculated from $\theta=\log ([$ BPP_1 $])$ curve are: $f=-7.59 ; K=4.66 \times 10^{6}$ and $\Delta G_{\mathrm{ads}}^{\circ}=-48.75 \mathrm{~kJ} \cdot \mathrm{mol}^{-1}$.

It is well known that values of $\Delta G_{\text {ads }}^{\circ}>-40 \mathrm{~kJ} \mathrm{~mol}^{-1}$ (Table 7), indicate a chemisorption mechanism. In addition to electrostatic interaction, there may be some other interactions [59]. The high $K_{\text {ads }}$ and $\Delta G_{\text {ads }}^{\circ}$ values may be attributed to higher adsorption of the inhibitor molecules at the metal-solution interface [60]. Moreover, the inhibition of copper by BPP compounds is often explained by the formation of $\mathrm{Cu}$ (II)-BPP through its heteroatoms [61]. A plot of IE\% versus Log ([BPP]) substituents has the character of an S-shape adsorbed isotherm as shown in Fig. 10. In physisorption process, it is assumed that acid anions such as $\mathrm{NO}_{3}{ }^{-}$ions are specifically adsorbed on the metal surface, donating an excess negative charge to the metal surface. In this way, potential of zero charge becomes less negative which promotes the adsorption of inhibitors in cationic form [62]; those of order of $40 \mathrm{~kJ} \mathrm{~mol}^{-1}$ or higher involve charge sharing or transfer from the inhibitor molecules to the metal surface to form a coordinate type of bond (chemisorption) [63]. The strong correlation coefficients of the fitted curves, reveals that the inhibition tendency of the inhibitors is due to the adsorption of these synthesized molecules on the metal surface [64], as given in Table 7. The slopes of $\ln (\theta / 1-\theta) \mathrm{C}$ versus $\theta$ plots are close to $\equiv 7-18$ in case of nitric acid solution which indicates the ideal simulating and expected from Frumkin adsorption isotherm [64]. $K_{\text {ads }}$ values were calculated from the intercepts of the straight lines on the $\ln (\theta / 1-\theta) \mathrm{C}$ [65]. Generally, the relatively high values of the adsorption equilibrium constant $\left(K_{\mathrm{ads}}\right)$ as shown in Table 7 , reflect the 
Fig. 9 Frumkin isotherm copper surface in $2.0 \mathrm{M} \mathrm{HNO}_{3}$ solution adsorption of (BPP_1) on the

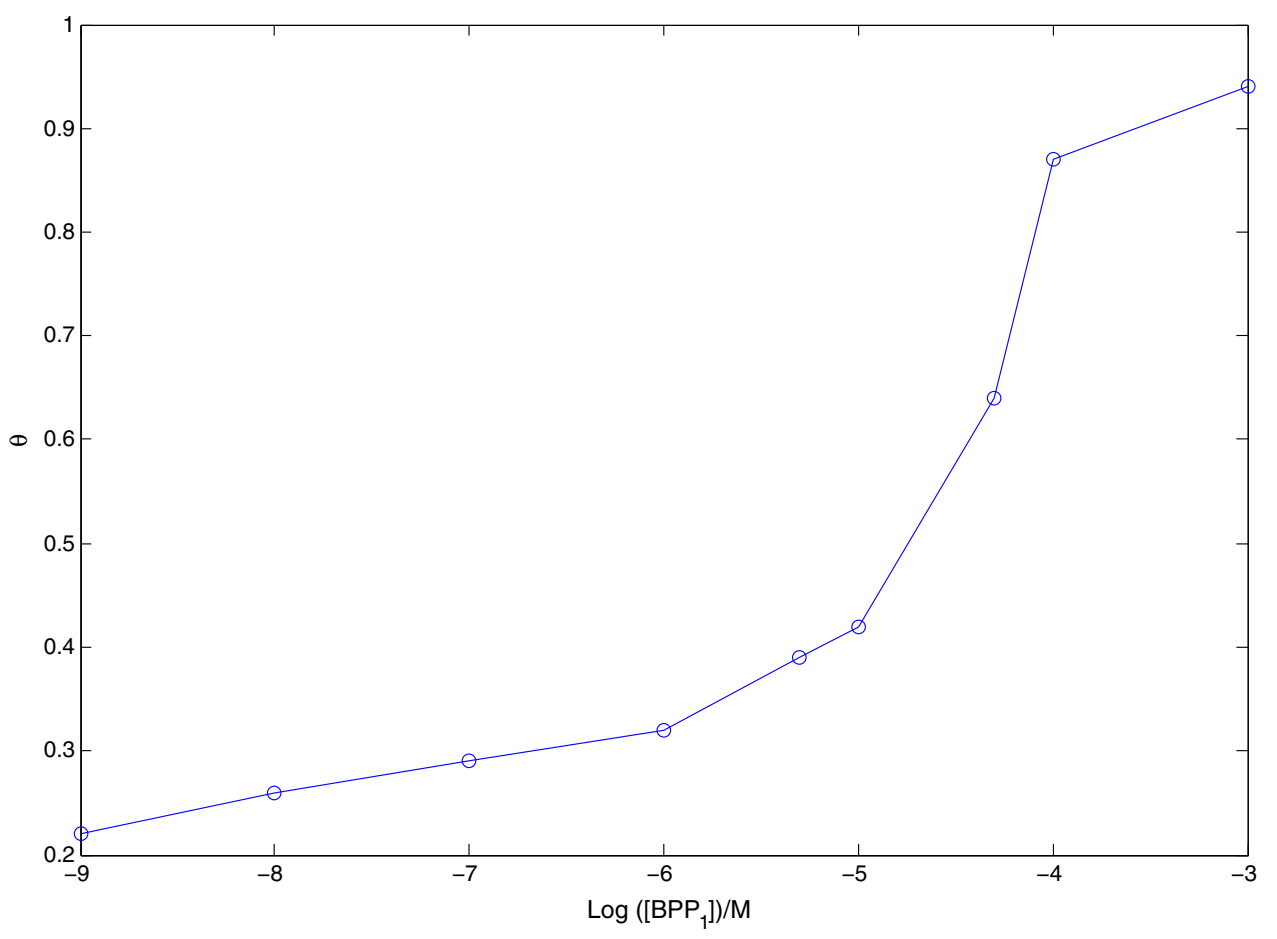

Table 7 Binding constant $\left(K_{\mathrm{b}}\right)$, active sites $(1 / y)$, lateral interaction $(f)$, equilibrium constant $\left(K_{\text {ads }}\right)$ and standard free energy of adsorption $\left(\Delta G^{\circ}\right)$ for copper in $2.0 \mathrm{M} \mathrm{HNO}_{3}$ for benzimidazole (BPP_1-7) molecules at $303 \mathrm{~K}$

\begin{tabular}{|c|c|c|c|c|c|c|}
\hline \multirow[t]{2}{*}{ BPP_1-7 inhibitors } & \multicolumn{3}{|c|}{ Kinetic model } & \multicolumn{3}{|c|}{ Frumkin adsorption isotherm } \\
\hline & $1 / y$ & $K_{\mathrm{b}}$ & $-\Delta G^{\circ}(\mathrm{kJ} / \mathrm{mol})$ & $-f$ & $K_{\text {ads }}$ & $-\Delta G_{\mathrm{ads}}^{\circ}(\mathrm{kJ} / \mathrm{mol})$ \\
\hline BPP_1 & 1.88 & 499.39 & 25.75 & 7.59 & $4.66 \times 10^{6}$ & 48.75 \\
\hline BPP_2 & 2.62 & 92.73 & 21.51 & 7.08 & $4.05 \times 10^{6}$ & 48.40 \\
\hline BPP_3 & 1.77 & 570.29 & 26.08 & 6.83 & $2.20 \times 10^{6}$ & 46.87 \\
\hline BPP_4 & 3.14 & 16.43 & 17.16 & 3.37 & $1.16 \times 10^{5}$ & 39.46 \\
\hline BPP_5 & 2.12 & 104.04 & 21.80 & 5.13 & $2.53 \times 10^{5}$ & 41.42 \\
\hline BPP_6 & 5.78 & 6.23 & 14.72 & 18.50 & $4.28 \times 10^{8}$ & 60.13 \\
\hline BPP_7 & 5.88 & 4.99 & 14.16 & 18.58 & $1.56 \times 10^{8}$ & 57.60 \\
\hline
\end{tabular}

high adsorption ability [66] of the BPP_1-7 molecules on the copper surface.

The corrosion inhibition mechanism for the BPP inhibitors on $\mathrm{Cu}$ surface in $\mathrm{HNO}_{3}$ is confirmed by finding the correlation between the kinetic parameters or the generalized mechanistic scheme of the kinetic-thermodynamic model proposed by El-Awady et al. [67, 68] (Eq. 30) with the experimental Frumkin adsorption isotherm.

$\theta /(1-\theta)=K^{\prime}[I]^{y} \quad$ or $\quad \log (\theta / 1-\theta)=\log K^{\prime}+y \log [I]$

where $y$ is the number of inhibitors molecules [I] occupying one active site, and $K^{\prime}$ is a constant, the relationship in Eq. (30) gives a satisfactory linear relation. Hence, the suggested model fits the obtained experimental data. The slope of such lines is the number of inhibitor molecules occupying a single active site, $(y)$ and the intercept is the binding constant $\left(\log K^{\prime}\right)$. As mentioned, $1 / y$ gives the number of active sites occupied by a single organic molecule and $K^{\prime y}$ is the equilibrium constant for the adsorption process. The binding constant $\left(K_{\mathrm{b}}\right)$ corresponding to that obtained from the known adsorption isotherms curve fitting is given by the following equation:

$K_{\mathrm{b}}=K^{\prime(1 / y)}$

Table 7 comprises the values of $1 / y$ and $K_{\mathrm{b}}$ for the studied BPP. This table show that the number of active sites occupied by one molecule in the case of BPP (1/ $y \equiv 2-6$ ). Values of $1 / y$ greater than unity implies the formation of multilayer of the inhibitor molecules on the 
Fig. 10 Variation of inhibition efficiency IE\% on copper with different concentrations of BPP_1-7 inhibitors in $2.0 \mathrm{M}$ $\mathrm{HNO}_{3}$ at $303 \mathrm{~K}$ for $4 \mathrm{~h}$ immersion time

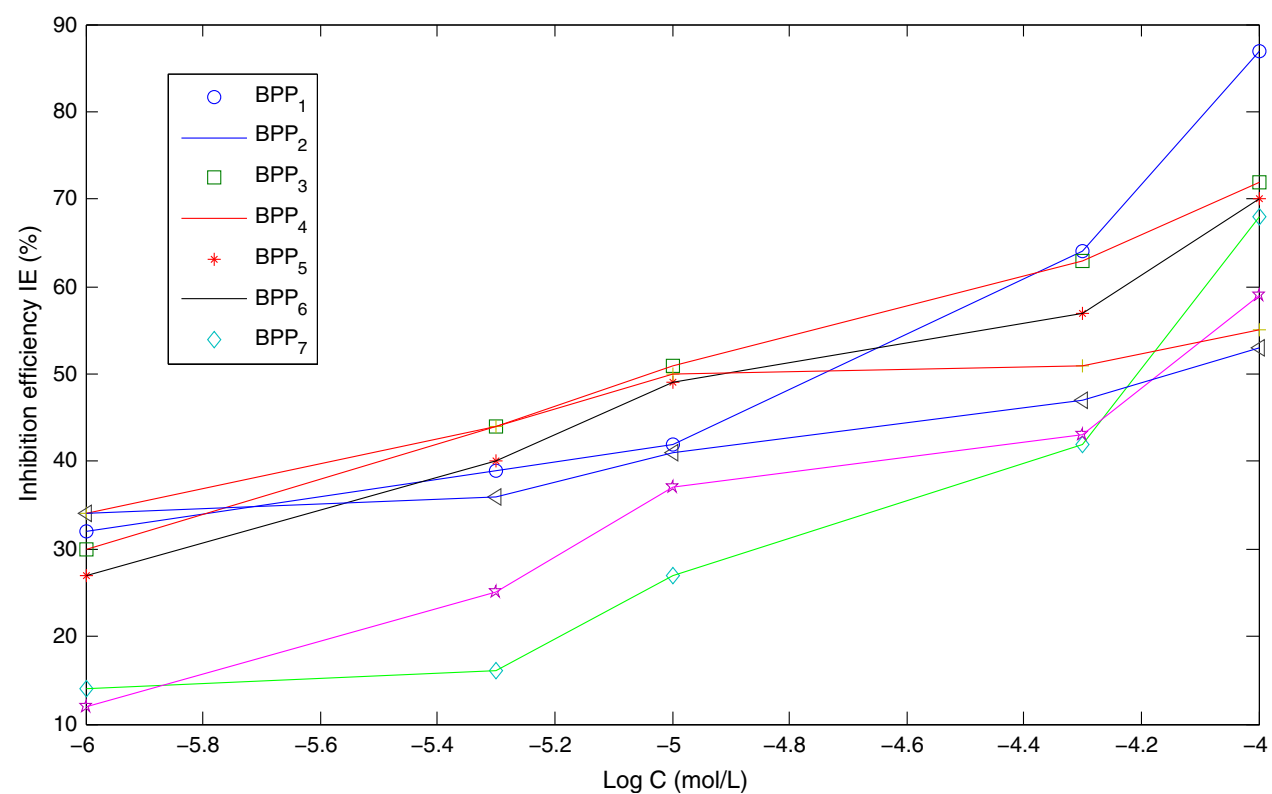

metal surface, whereas, values of $1 / y$ less than unity indicates that a given inhibitor molecule will occupy more than one active site [68]. According to the proposed kineticthermodynamic model, the adsorption takes place via formation of multilayer of the inhibitor molecules on the copper electrode surface. The slope values do not equal unity (gradient slopes $<1$ ); hence, the adsorption of these synthesized BPP on copper surface does not obey a Langmuir adsorption isotherm. Frumkin adsorption isotherm (Eq. 28) represents best fit for experimental data obtained from applying BPP as chemical inhibitors on copper in $2.0 \mathrm{M} \mathrm{HNO}_{3}$ acid solution. The values of $K_{\text {ads }}$ (equilibrium constant of the inhibitor adsorption process) and $(f)$ are tabulated in Table 7 . The lateral interaction parameter $(f)$ has negative values; this parameter is a measure of the degree of steepness of the adsorption isotherm. The adsorption equilibrium constant $\left(K_{\text {ads }}\right)$ calculated from Frumkin equation acquires lower values than those binding constant $\left(K_{\mathrm{b}}\right)$ obtained and calculated from the kinetic-thermodynamic model. The lack of compatibility of the calculated $\left(K_{\mathrm{b}}\right)$ and experimental $\left(K_{\mathrm{ads}}\right)$ values may be attributed to the fact that Temkin adsorption isotherm is only applicable to cases where one active site per inhibitor molecule is occupied. The lateral interaction parameter was introduced to treat of deviations from Langmuir ideal behavior, whereas the kinetic-thermodynamic model uses the size parameter. The values of the lateral interaction parameter $(-f)$ were found to be negative and increase from $\equiv 6.83$ to 18.58 . This denotes that an increase in the adsorption energy takes place with the increase in the surface coverage $(\theta)$. Adsorption process is a displacement reaction involving removal of adsorbed water molecules from the electrode metal surface and their substitution by inhibitor molecules. Thus, during adsorption, the adsorption equilibrium forms an important part in the overall free energy changes in the process of adsorption. It has been shown that, the free energy change $\left(\Delta G_{\mathrm{ads}}^{\circ}\right)$ increases with increase of the solvating energy of adsorbing species, which in turn increases with the size of hydrocarbon portion in the organic molecule and the number of active sites. Hence, the increase of the molecular size leads to decreased solubility, and increased absorbability. The large negative values of the standard free energy changes of adsorption $\left(\Delta G_{\text {ads }}^{\circ}\right)$, obtained for BPP, indicate that the reaction is proceeding spontaneously and accompanied with a high efficient adsorption. Although, the obtained values of the binding constant $\left(K_{\mathrm{b}}\right)$ from the kinetic model and the modified equilibrium constant $\left(K_{\mathrm{ads}}\right)$ from Temkin equation are incompatible, generally have large values (Table 7), mean better inhibition efficiency of the investigated synthesized BPP_1-7, i.e., stronger electrical interaction between the double layer existing at the phase boundary and the adsorbing molecules. In general, the equilibrium constant of adsorption $\left(K_{\mathrm{ads}}\right)$ was found to become higher with increasing the inhibition efficiency of the inhibitor studied as given in Table 7. Application of kinetic-thermodynamic model on benzimidazole (BPP_1-7) inhibitors of copper in $2.0 \mathrm{M} \mathrm{HNO}_{3}$ at $303 \mathrm{~K}$ is shown in Fig. 11.

\section{Effect of temperature}

Temperature has a great effect on the rate of metal electrochemical corrosion. In case of corrosion in a neutral 

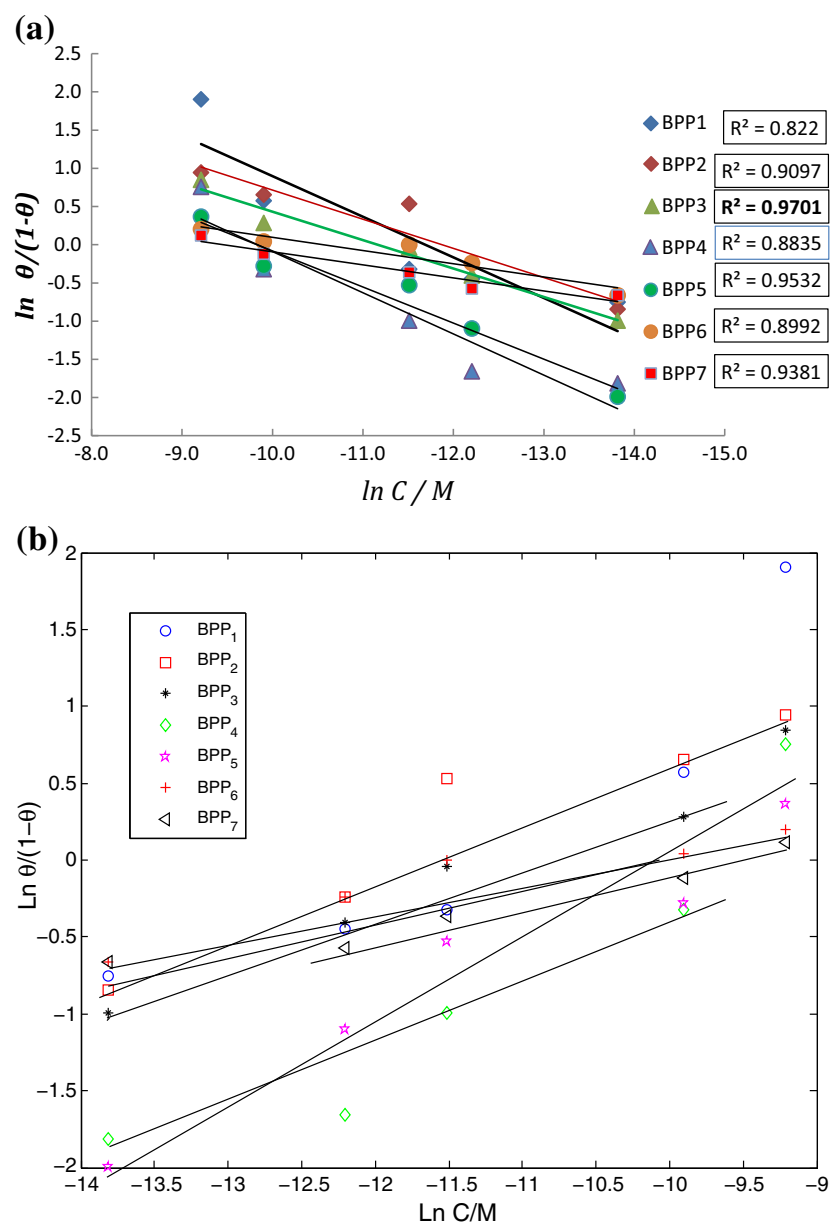

Fig. 11 Application of kinetic-thermodynamic model on benzimidazole (BPP_1-7) inhibitors of copper in $2.0 \mathrm{M} \mathrm{HNO}_{3}$ at $303 \mathrm{~K}$

solution (oxygen depolarization), the increase in temperature has a favourable effect on the overpotential of oxygen depolarization and the rate of oxygen diffusion but it leads to a decrease of oxygen solubility. In case of corrosion in acidic medium (hydrogen depolarization), the corrosion rate increases exponentially with temperature increase because the hydrogen evolution over potential decreases [69]. The potentiodynamic polarization curves for copper corrosion in $2.0 \mathrm{M} \mathrm{HNO}_{3}$ acid in the presence of $1 \times 10^{-6}$ M BBP_1 at temperatures $30,40,50$ and $60{ }^{\circ} \mathrm{C}$ is given in Fig. 12.

The relationship between the corrosion rate $\left(R_{\text {corr }}\right)$ of copper in acidic media and temperature $(T)$ is often expressed by the Arrhenius equation [70, 71]:

$\ln R_{\text {corr }}=\ln A-\frac{E_{\mathrm{a}}}{\mathrm{RT}}$

where $E_{\mathrm{a}}$ is the apparent activation energy, $R$ is the molar gas constant $\left(8.314 \mathrm{~J} \mathrm{~K}^{-1} \mathrm{~mol}^{-1}\right), T$ is the absolute temperature, and $A$ is the frequency factor. The plot of $\operatorname{Ln} R_{\text {corr }}$

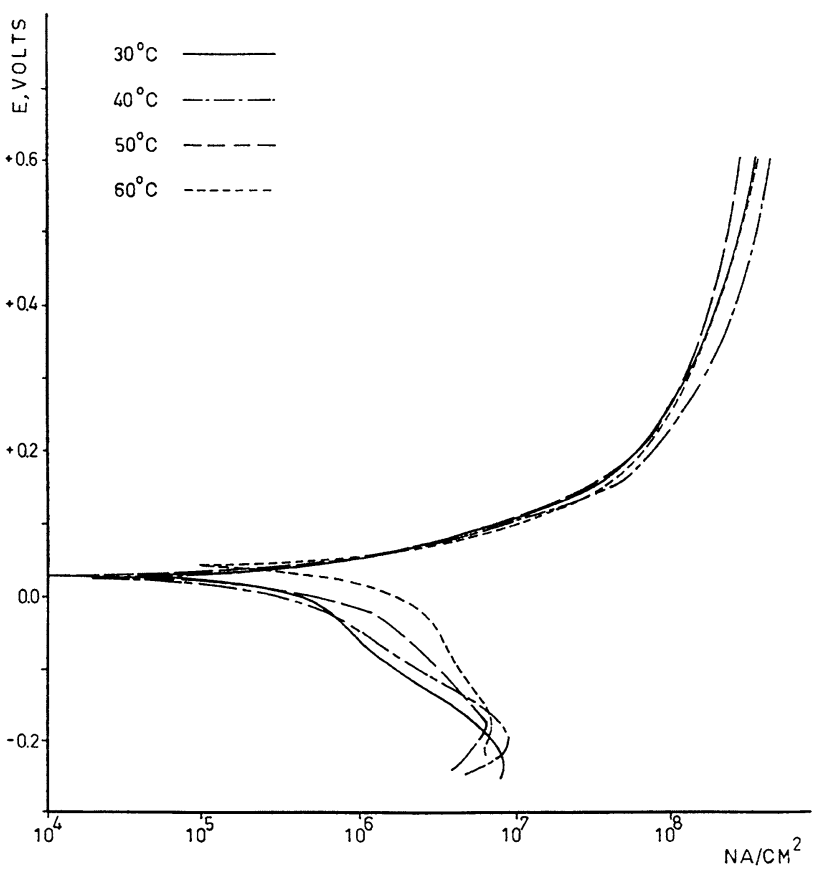

Fig. 12 Potentiodynamic polarization curves of copper in $2.0 \mathrm{M}$ $\mathrm{HNO}_{3}$ solution with the $1 \times 10^{-6} \mathrm{M}$ of BPP_1 at different temperatures

against $1 / T$ for copper corrosion in $2.0 \mathrm{M} \mathrm{HNO}_{3}$ in the absence and presence of different concentrations of BPP is shown in Fig. 13. All parameters were given in Table 8. The activation energy increased in the presence of BPP, which indicated physical (electrostatic) adsorption. Furthermore, the activation energy rose with increasing inhibitor concentration, suggesting strong adsorption of inhibitor molecules at the metal surface [60]. The increase in activation energy was due to the corrosion reaction mechanism in which charge transfer was blocked by the adsorption of BPP molecules on the copper surface [72]. It also revealed that the whole process was controlled by the surface reaction since the energy of the activation corrosion process in both the absence and presence of BPP was greater than $20 \mathrm{~kJ} \mathrm{~mol}^{-1}$ [73].

Experimental corrosion rate values obtained from Potentiodynamic polarization measurements for copper in $2.0 \mathrm{M} \mathrm{HNO}_{3}$ in the absence and presence of BPP was used to further gain insight on the change of enthalpy $\left(\Delta H^{*}\right)$ and entropy $\left(\Delta S^{*}\right)$ of activation for the formation of the activation complex in the transition state using transition equation [63]:

$R_{\text {corr }}=\frac{\mathrm{RT}}{N h} \exp \frac{\left(\Delta S^{*}\right)}{R} \exp \left(-\frac{\Delta H^{*}}{\mathrm{RT}}\right)$

where $h$ is the Plank's constant $\left(6.626176 \times 10^{-34} \mathrm{~J} \mathrm{~s}\right), N$ is the Avogadro's number $\left(6.02252 \times 10^{-23} \mathrm{~mol}^{-1}\right), R$ is the universal gas constant and $T$ is absolute temperature. 
Fig. 13 Arrhenius plots of Ln $R_{\text {corr }}$ versus $1 / \mathrm{T}$ for copper in $2.0 \mathrm{M} \mathrm{HNO}_{3}$ in the absence and the presence of BPP_1 at optimum concentration

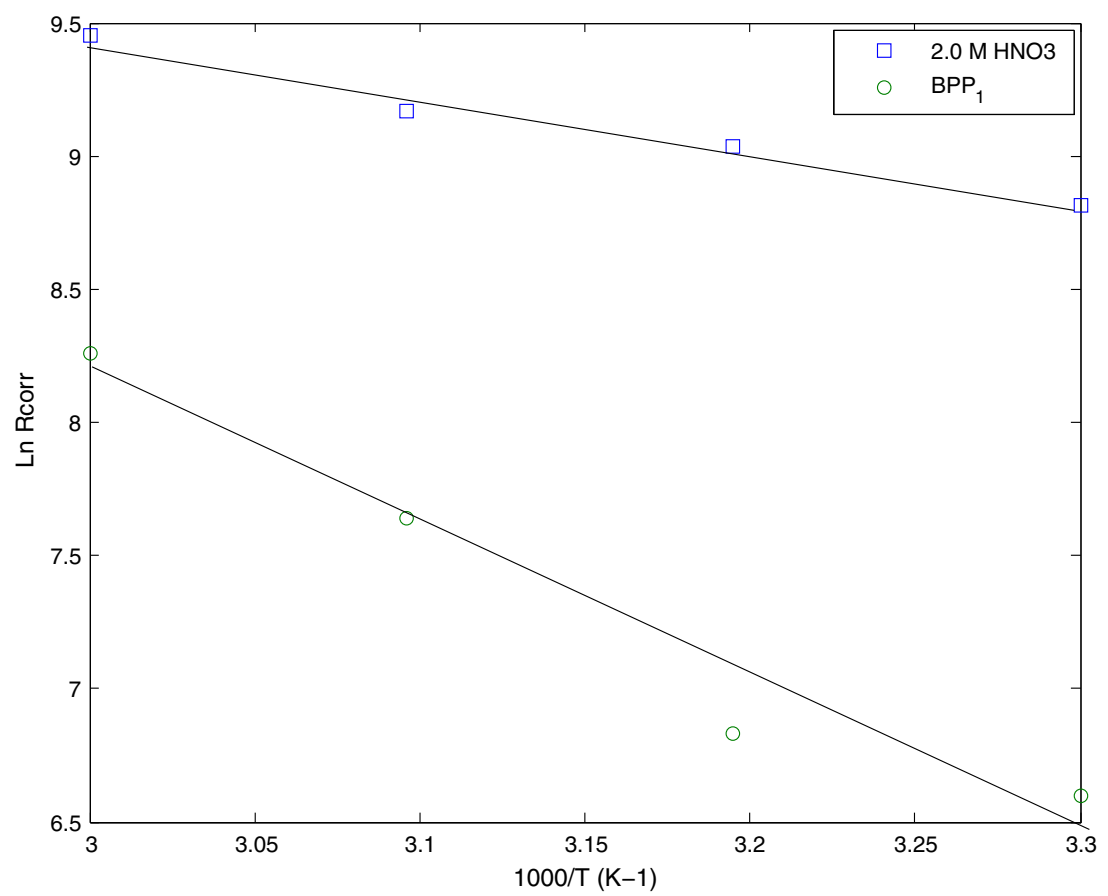

The free energy change of activation $\left(\Delta G^{*}\right)$ is obtained from the Eyring equation: (Eq. (34).

$k=\frac{k_{\mathrm{B}} T}{h} \mathrm{e}^{-\Delta G^{*} / \mathrm{RT}}$

Another convenient form of Eq. (34) is Eq. (35).

$\Delta G^{*}=\Delta H^{*}-T \Delta S^{*}$

The relation of $\operatorname{Ln} R_{\text {corr }} / T$ versus $1 / T$ for copper corrosion in $2.0 \mathrm{M} \mathrm{HNO}_{3}$ in the absence and presence of different concentrations of BPP is given in Fig. 14. Straight lines were obtained with slope of $\left(\Delta H^{*} / R\right)$ and an intercept of $\left[\operatorname{Ln}(R / N h)+\left(\Delta S^{*} / R\right)\right]$ from which the values of $\Delta H^{*}$ and $\Delta S^{*}$, respectively, were computed.

The activation parameters were computed and listed in Table 9. Inspection of these data reveal that the activation parameters $\left(E_{\mathrm{a}}, \Delta H^{*}, \Delta S^{*}\right.$ and $\left.\Delta G^{*}\right)$ of dissolution reaction of copper in $2.0 \mathrm{M} \mathrm{HNO}_{3}$ in the presence of BPP are higher than in the absence of inhibitor. The positive sign of the enthalpy of activation reflect the endothermic nature of copper dissolution process meaning that dissolution of copper is difficult [74]. The entropy of activation was negative in absence of BPP, whereas $\Delta S^{*}$ was changed between negative and positive values in presence of BPP implying that the ratedetermining step for the activated complex is dissociation step rather than association. In other words, the adsorption process is accompanied by an increase in entropy, which is the driving force for the adsorption of inhibitor onto the copper surface [75]. From the thermodynamic parameters in Table 9, it can be seen that $E_{\mathrm{a}}$ increases as the inhibition efficiency of the additives increases. This suggests that the process is controlled by a surface reaction, since the energy of activation for the corrosion process is above $20 \mathrm{~kJ} \mathrm{~mol}^{-1}$. The higher entropy values $\left(\Delta S^{*}\right)$ as shown in Table 9 in the case of different inhibitors compared to a high negative values of $\left(-121.16 \mathrm{~J} . \mathrm{K}^{-1} \mathrm{~mol}^{-1}\right)$ in the case of free nitric acid solution indicates a slower reaction [76]. The negative entropy $\left(\Delta S^{*}\right)$ values only in the case of BPP_1 and BPP_4 inhibitors, with their lower activation energy $\left(E_{\mathrm{a}}\right)$ values indicate that the copper corrosion inhibition process in these derivatives only is entropy controlled rather than activation energy controlled (Table 9). The copper corrosion inhibition process in the case of all other BPP inhibitors is activation energy controlled, since the activation energy $\left(E_{\mathrm{a}}\right)$ values are relatively high, as shown in Table 9 . The activation energy $\left(E_{\mathrm{a}}\right)$ values support the sequence arrangement of different inhibitors according to their increasing inhibition efficiency (IE\%) values. The activation energy for copper was found between 2, 4.6 and11.7 $\mathrm{kcal} \mathrm{mol}^{-1}$ in $0.1 \mathrm{M} \mathrm{HCI}, 4.0 \mathrm{M}$ $\mathrm{HNO}_{3}$ and $3.0 \mathrm{M} \mathrm{HNO}_{3}$ solutions, respectively. Generally, one can say that the nature and concentration of the electrolyte greatly affect the activation energy for the corrosion process.

\section{Theoretical study}

\section{Quantum chemical calculations}

For the purpose of determining the active sites of the inhibitor molecule, three influence factors: natural atomic 
Table 8 Various corrosion parameters for copper in $2.0 \mathrm{M} \mathrm{HNO}_{3}$ in absence and presence of optimum concentration $1 \times 10^{-6} \mathrm{M}$ of benzimidazole (BPP_ 1-7) at different temperatures, $303 \leq T \leq 333 \mathrm{~K}$

\begin{tabular}{|c|c|c|c|c|c|c|c|c|}
\hline System & Temperature (K) & $R_{\mathrm{p}}\left(\Omega \mathrm{cm}^{2}\right)$ & $I_{\text {corr }}\left(\mu \mathrm{A} \mathrm{cm}^{-2}\right)$ & $R_{\text {corr }}(\mathrm{MPY})$ & $E_{\text {corr }} \mathrm{mV}(\mathrm{SCE})$ & $\beta_{\mathrm{c}}\left(\mathrm{V} \mathrm{dec}{ }^{-1}\right)$ & $\beta_{\mathrm{a}}\left(\mathrm{V} \mathrm{dec}^{-1}\right)$ & $\mathrm{IE} \%$ \\
\hline \multirow[t]{4}{*}{$2.0 \mathrm{M} \mathrm{HNO}_{3}$} & 303 & 6.360 & 7287 & 6719 & 29 & 0.302 & 0.165 & - \\
\hline & 313 & 5.433 & 10,180 & 8384 & 49 & 0.366 & 0.195 & - \\
\hline & 323 & 4.577 & 10,380 & 9570 & 43 & 0.250 & 0.195 & - \\
\hline & 333 & 3.001 & 13,860 & 12,770 & 45 & 0.170 & 0.219 & - \\
\hline \multirow{4}{*}{$\begin{array}{l}2.0 \mathrm{M} \\
\mathrm{HNO}_{3}+\text { BPP_1 }\end{array}$} & 303 & 45.08 & 235 & 731.3 & 23 & 0.177 & 0.154 & 96.8 \\
\hline & 313 & 37.14 & 1004 & 926.1 & 26 & 0.178 & 0.166 & 90.1 \\
\hline & 323 & 30.30 & 2257 & 2080 & 25 & 0.244 & 0.186 & 78.2 \\
\hline & 333 & 14.50 & 4194 & 3866 & 39 & 0.381 & 0.222 & 69.7 \\
\hline \multirow{3}{*}{$\begin{array}{l}2.0 \mathrm{M} \\
\mathrm{HNO}_{3}+\text { BPP_2 }\end{array}$} & 303 & 75.34 & 406.7 & 374.9 & 12 & 0.162 & 0.125 & 94.4 \\
\hline & 313 & 32.96 & 1399 & 1289 & 21 & 0.132 & 0.186 & 86.2 \\
\hline & 323 & 74.83 & 2807 & 8119 & 41 & 0.335 & 0.212 & 72.9 \\
\hline \multirow{4}{*}{$\begin{array}{l}2.0 \mathrm{M} \\
\mathrm{HNO}_{3}+\text { BPP_3 }\end{array}$} & 303 & 63.36 & 507 & 467.4 & 7 & 0.145 & 0.151 & 93.0 \\
\hline & 313 & 26.35 & 1647 & 1518 & 21 & 0.220 & 0.183 & 83.8 \\
\hline & 323 & 25.80 & 2100 & 3567 & 23 & 0.215 & 0.190 & 79.7 \\
\hline & 333 & 50.67 & 4190 & 11,240 & 44 & 0.333 & 0.248 & 69.7 \\
\hline \multirow{4}{*}{$\begin{array}{l}2.0 \mathrm{M} \\
\qquad \mathrm{HNO}_{3}+\text { BPP_4 }\end{array}$} & 303 & 30.93 & 1180 & 1206 & 26 & 0.185 & 0.188 & 83.8 \\
\hline & 313 & 24.13 & 1766 & 1546 & 17 & 0.187 & 0.186 & 82.6 \\
\hline & 323 & 13.83 & 2916 & 2688 & -34 & 0.162 & 0.219 & 71.9 \\
\hline & 333 & 7.026 & 7287 & 6718 & 35 & 0.252 & 0.222 & 47.4 \\
\hline \multirow{3}{*}{$\begin{array}{l}2.0 \mathrm{M} \\
\mathrm{HNO}_{3}+\text { BPP_5 }\end{array}$} & 303 & 76.40 & 397.2 & 366.2 & 7 & 0.202 & 0.107 & 94.5 \\
\hline & 313 & 50.24 & 700.6 & 645.9 & 15 & 0.185 & 0.144 & 93.1 \\
\hline & 323 & 14.05 & 3067 & 2827 & 26 & 0.193 & 0.204 & 70.4 \\
\hline \multirow{3}{*}{$\begin{array}{l}2.0 \mathrm{M} \\
\mathrm{HNO}_{3}+\text { BPP_6 }\end{array}$} & 303 & 147.9 & 320 & 295.1 & 6 & 0.204 & 0.234 & 95.6 \\
\hline & 313 & 20.81 & 2440 & 344 & 25 & 0.402 & 0.165 & 76.0 \\
\hline & 323 & 15.72 & 3590 & 2249 & 30 & 0.302 & 0.228 & 65.4 \\
\hline \multirow{4}{*}{$\begin{array}{l}2.0 \mathrm{M} \\
\qquad \mathrm{HNO}_{3}+\text { BPP_7 }\end{array}$} & 303 & 113.0 & 242.5 & 290 & -3 & 0.124 & 0.129 & 96.6 \\
\hline & 313 & 46.8 & 724.7 & 668.1 & 8 & 0.139 & 0.179 & 92.9 \\
\hline & 323 & 9.743 & 4087 & 4690 & 30 & 0.213 & 0.245 & 60.6 \\
\hline & 333 & 9.410 & 6575 & 5140 & 30 & 0.236 & 0.229 & 52.5 \\
\hline
\end{tabular}

charge, distribution of frontier orbital, and Fukui indices are considered. According to classical chemical theory, all chemical interactions are by either electrostatic or orbital. Electrical charges in the molecule were obviously the driving force of electrostatic interactions it has been proven that local electron densities or charges are important in many chemical reactions and physico-chemical properties of compounds [30]. The inhibition efficiencies of seven benzimidazole (BPP_1-7) derivatives on the corrosion of copper were investigated by quantum chemical and molecular dynamics simulation studies.

The molecular reactivity of the studied molecules was investigated and compared via analysis of frontier molecular orbitals. The energy of HOMO is associated with the electron donating ability of a molecule. High values of energy of HOMO state that the molecule is prone to donate electrons to appropriate acceptor molecules with low- energy and empty molecular orbital. On the other hand, LUMO energy level is an indicator of electron accepting abilities of molecules. It is important to note that the molecules that have lower LUMO energy value have more electron accepting ability. The reactive ability of the inhibitor is considered to be closely related to their frontier molecular orbitals, the HOMO and LUMO. The frontier molecule orbital density distributions involving the optimized structures, HOMOs, LUMOs with Mulliken orbital charges population's analyses of the investigated seven (BPP_1-7) molecules are presented in Figs. 15 and 16. The optimized structures, HOMOs, LUMOs of non-protonated inhibitor molecules (BPP_1-7) using DFT/B3LYP/6$31++\mathrm{G}(d, p)$ are shown in Fig. 15. As seen from the figure, the populations of the HOMO focused around the carbon chain containing imidazole and azo nitrogen. But the LUMO densities were mainly around the benzene 
Fig. 14 Arrhenius plots of Ln $R_{\text {corr }} / \mathrm{T}$ versus $1 / \mathrm{T}$ for copper in $2.0 \mathrm{M} \mathrm{HNO}_{3}$ in the absence and the presence of BPP_1 at optimum concentration

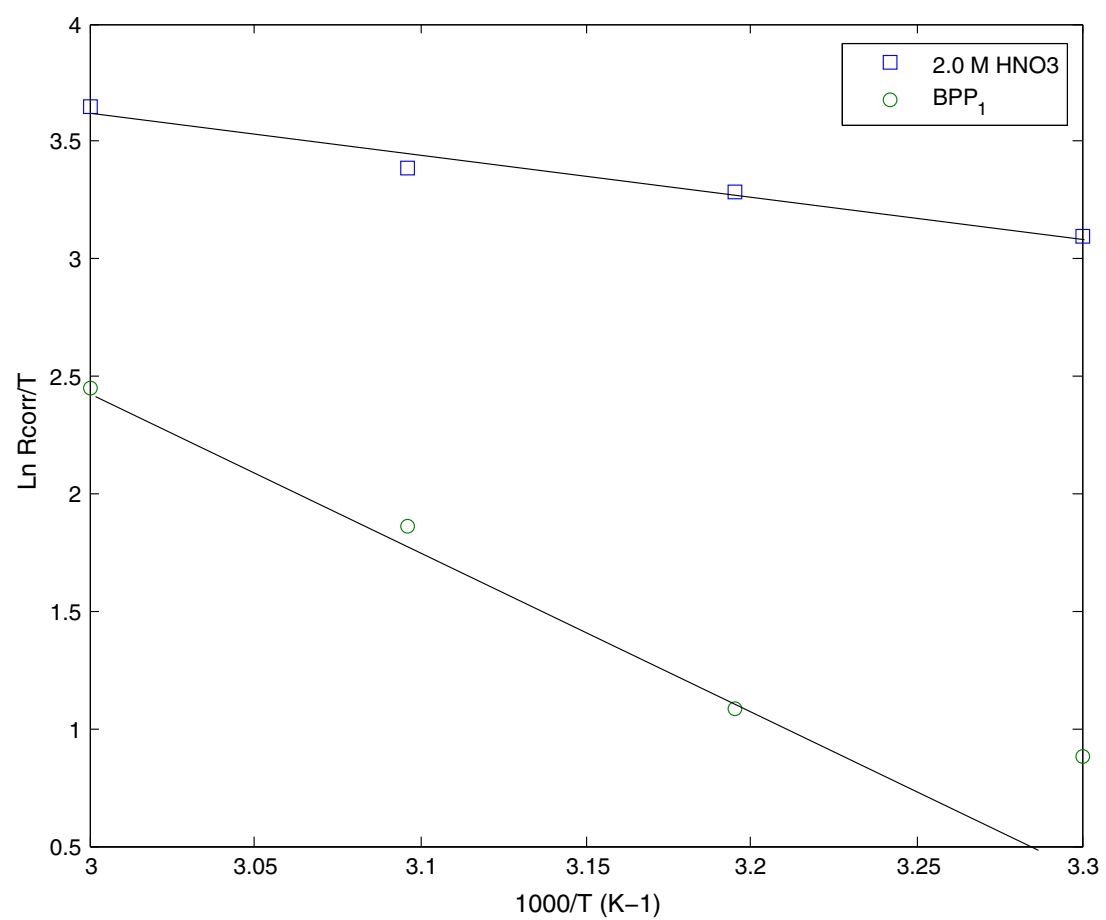

Table 9 Activation energy $\left(E_{\mathrm{a}}\right)$, enthalpy change $\left(\Delta H^{*}\right)$, free energy change $\left(\Delta G^{*}\right)$, and entropy change $\left(\Delta S^{*}\right)$ for the corrosion of copper in $2.0 \mathrm{M} \mathrm{HNO}_{3}$ in absence and presence of $1 \times 10^{-6} \mathrm{M}$ of benzimidazole (BPP_1-7) inhibitors at different temperatures, $303 \leq T \leq 333 \mathrm{~K}$

\begin{tabular}{llcccc}
\hline System & A (MPY) & $E_{\mathrm{a}}\left(\mathrm{kJ} \mathrm{mol}^{-1}\right)$ & $\Delta H^{*}\left(\mathrm{~kJ} \mathrm{~mol}^{-1}\right)$ & $\Delta G^{*}\left(\mathrm{~kJ} \mathrm{~mol}^{-1}\right)$ & $\Delta S^{*}\left(\mathrm{~J} \mathrm{~K}^{-1} \mathrm{~mol}^{-1}\right)$ \\
\hline $2.0 \mathrm{M} \mathrm{HNO}_{3}$ & $6.953 \times 10^{6}$ & 17.481 & 15.403 & 53.932 & -121.16 \\
$2.0 \mathrm{M} \mathrm{HNO}_{3}+$ BPP_1 & $4.883 \times 10^{11}$ & 51.632 & 46.473 & 58.608 & -38.161 \\
$2.0 \mathrm{M} \mathrm{HNO}_{3}+$ BPP_2 & $2.201 \times 10^{24}$ & 126.453 & 123.100 & 57.546 & 209.438 \\
$2.0 \mathrm{M} \mathrm{HNO}_{3}+$ BPP_3 & $1.225 \times 10^{18}$ & 89.407 & 85.280 & 57.482 & 87.413 \\
$2.0 \mathrm{M} \mathrm{HNO}_{3}+$ BPP_4 & $8.419 \times 10^{4}$ & 9.351 & 44.853 & 57.408 & -39.483 \\
$2.0 \mathrm{M} \mathrm{HNO}_{3}+$ BPP_5 & $1.683 \times 10^{17}$ & 85.419 & 80.922 & 59.107 & 69.696 \\
$2.0 \mathrm{M} \mathrm{HNO}_{3}+$ BPP_6 & $3.470 \times 10^{16}$ & 82.253 & 80.287 & 60.036 & 64.700 \\
$2.0 \mathrm{M} \mathrm{HNO}_{3}+$ BPP_7 & $9.336 \times 10^{17}$ & 89.883 & 86.928 & 58.67 & 88.860 \\
\hline
\end{tabular}

cyclic. Higher HOMO energy $\left(E_{\mathrm{HOMO}}\right)$ of the molecule means a higher electron-donating ability to appropriate acceptor molecules with low-energy empty molecular orbital and thus explains the adsorption on metallic surfaces by way of delocalized pairs of $\pi$-electrons. $E_{\mathrm{LUMO}}$, the energy of the lowest unoccupied molecular orbital, signifies the electron receiving tendency of a molecule. From Fig. 15, it could be seen that BPP_1-7 have similar HOMO and LUMO distributions, which were all located on the entire BPP moiety. This is due to the presence of nitrogen and oxygen atoms together with several $\pi$-electrons on the entire molecule. Thus, unoccupied d orbital of $\mathrm{Cu}$ atom can accept electrons from inhibitor molecule to form coordinate bond. Also the inhibitor molecule can accept electrons from $\mathrm{Cu}$ atom with its anti-bonding orbitals to form back donating bond. Figure 16 shows B3LYP/
6-311G** selected bond length for the optimized geometry of the studied compounds calculated for BPP. It has been reported that the more negative the atomic charges of the adsorbed center, the more easily the atom donates its electron to the unoccupied orbital of the metal $[14,30]$. Mulliken orbital charges populations analysis of BPP_ 1-7 derivatives using DFT method at the B3LYP method with $6-31+\mathrm{G}^{* *}$ basis sets level are presented in Fig. 16. It can be seen that the area of carbon bone chain containing imidazole and azo nitrogen, hydroxyl, and methyl charged a large electron density and might form adsorption active centers. It is clear from Fig. 16 that nitrogen and oxygen as well as some carbon atoms carry negative charge centers which could offer electrons to the copper surface to form a coordinate bond. This shows that the $\mathrm{N}$ and $\mathrm{O}$ atoms are the probable reactive sites for the adsorption of copper. Higher 


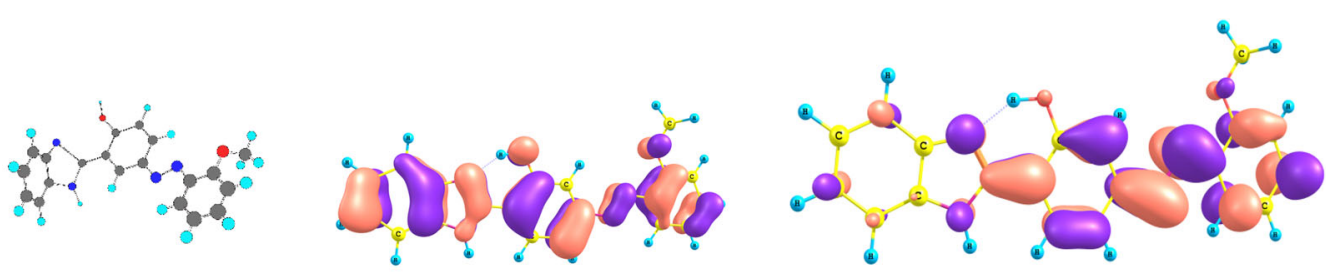

BPP_1
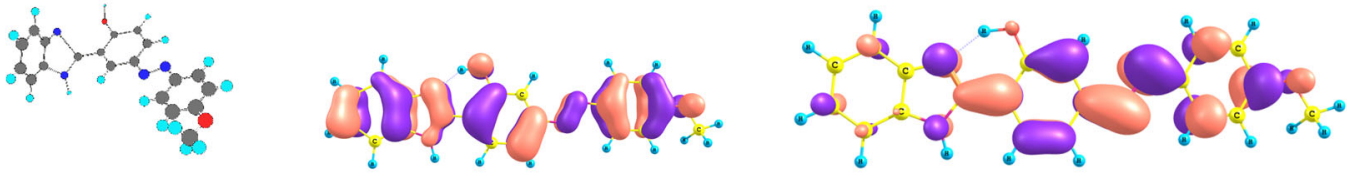

BPP_2
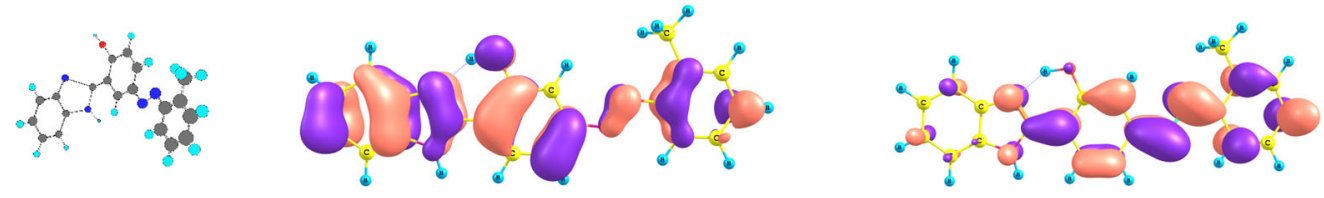

BPP_3
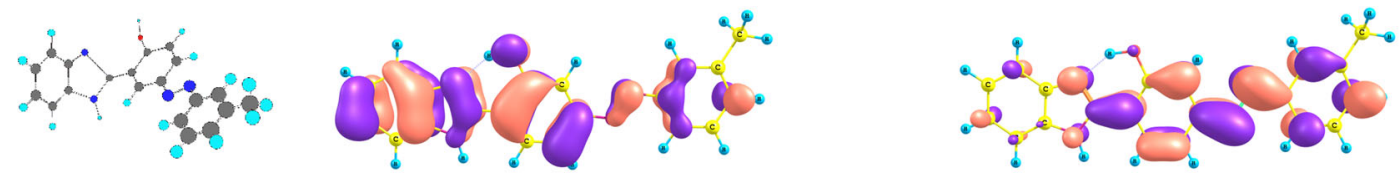

\section{BPP_4}
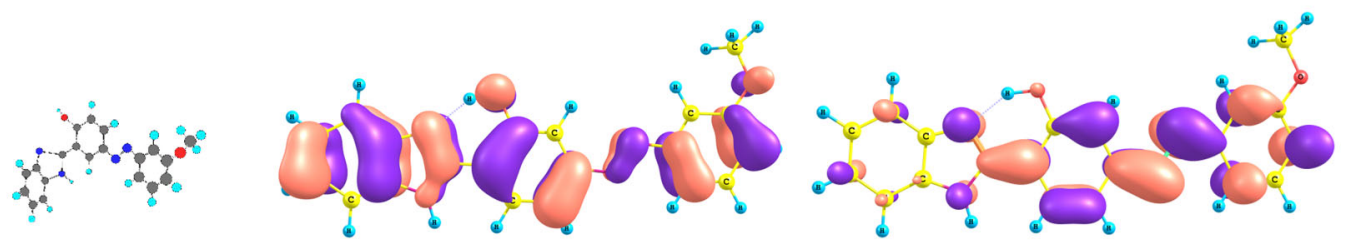

BPP 5
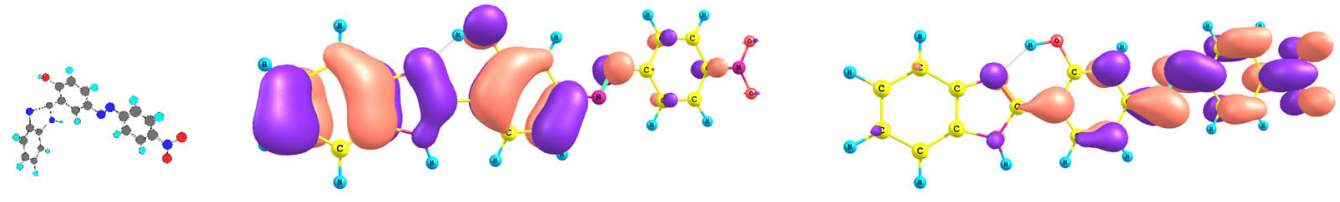

BPP_6
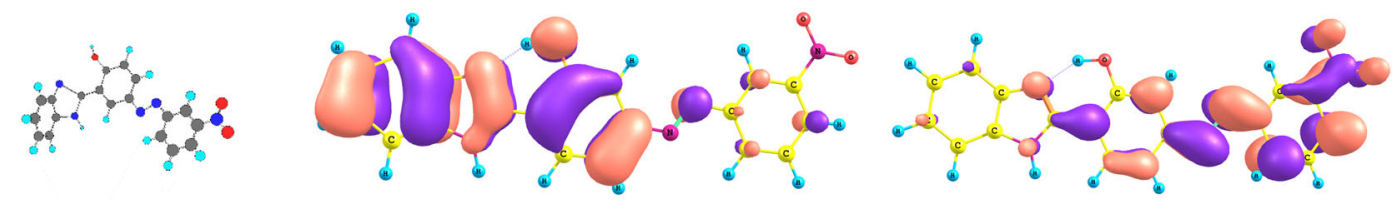

BPP_ 7

Structure

HOMO

LUMO

Fig. 15 The optimized structures, HOMOs, LUMOs of non-protonated inhibitor molecules (BPP_1-7) using DFT/B3LYP/6-31++G $(d, p)$ 
Fig. 16 Mulliken orbital charges populations analysis of BPP_1-7 derivatives using DFT method at the B3LYP method with $6-31+\mathrm{G}^{* *}$ basis sets level

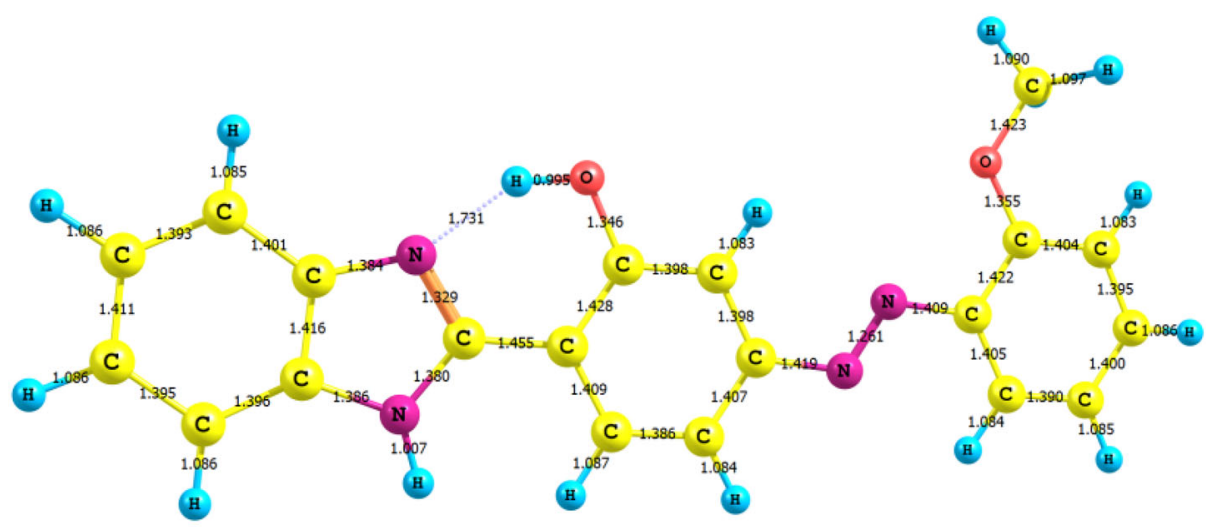

BPP 1

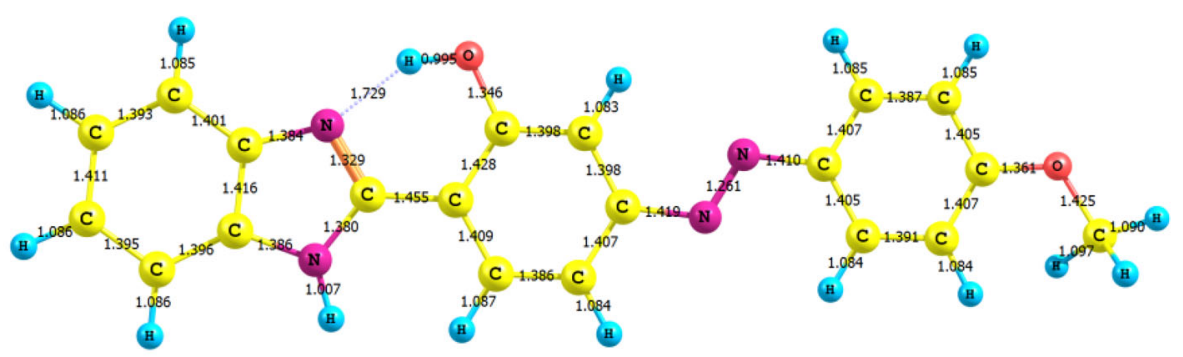

BPP_2

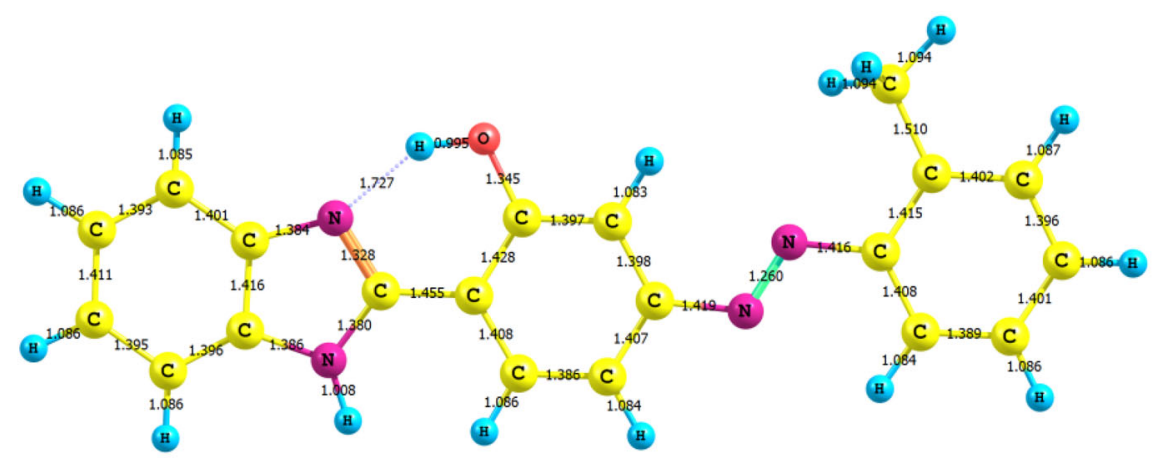

BPP_3

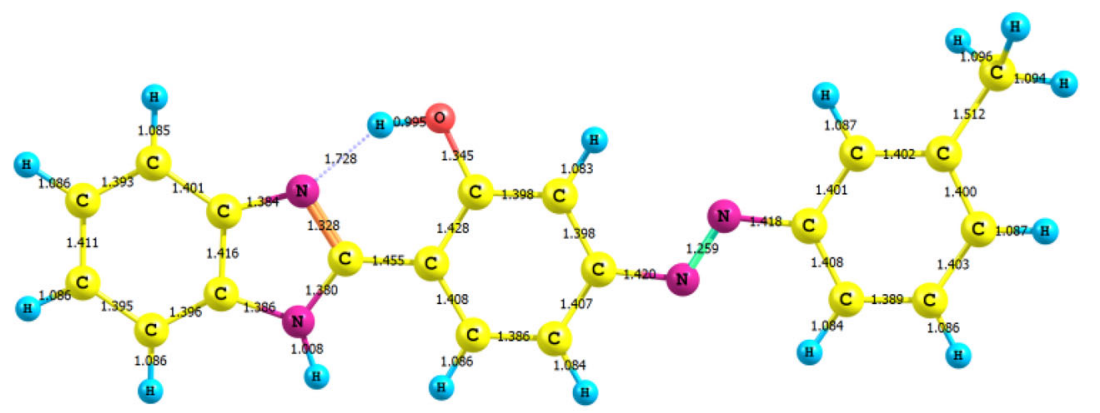

BPP_4 
Fig. 16 continued

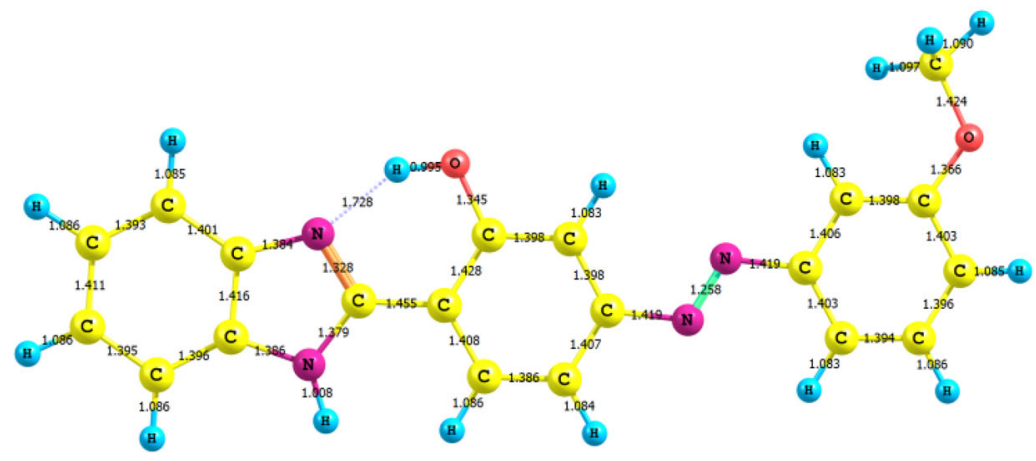

BPP_5

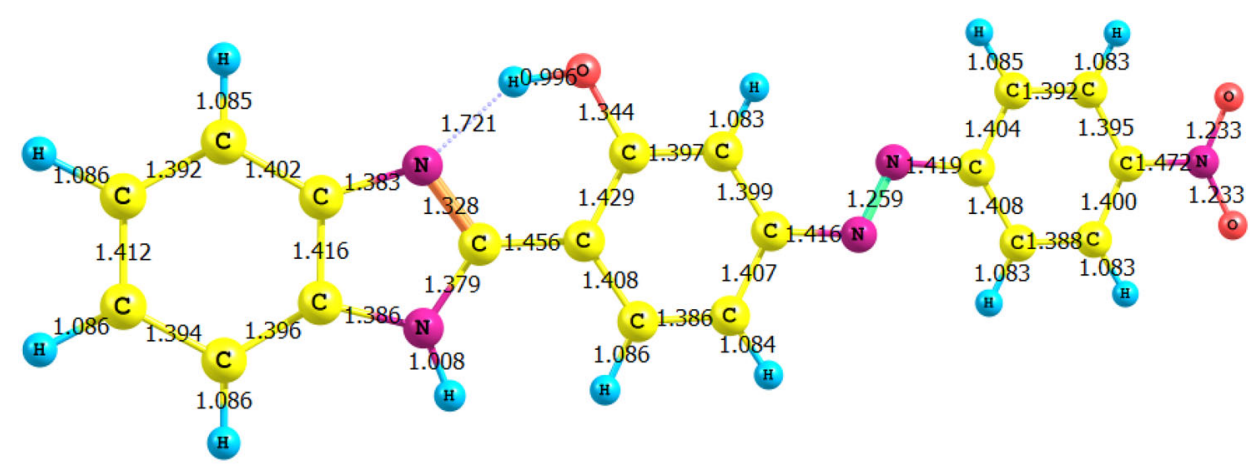

\section{BPP_6}

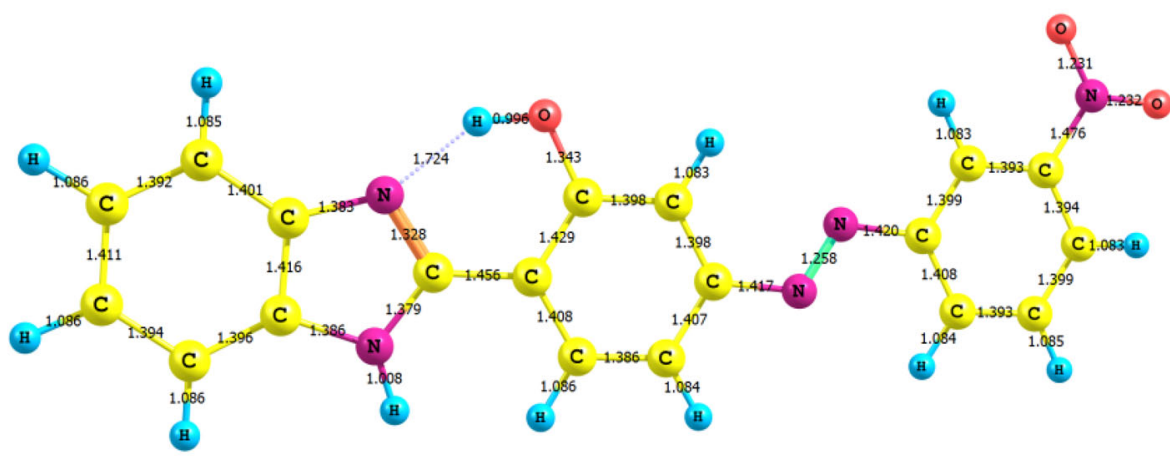

BPP_ 7

values of $E_{\mathrm{HOMO}}$ are likely to indicate a tendency of the molecule to donate electrons to appropriate acceptor molecules with low-energy or empty electron orbital. Quantum chemical parameters such as $E_{\mathrm{HOMO}}, E_{\mathrm{LUMO}}, \Delta \mathrm{E}$ (HOMO-LUMO energy gap), chemical hardness, softness, electronegativity, proton affinity, electrophilicity and nucleophilicity are important and useful tools to compare the corrosion inhibition performances of molecules. Calculated quantum chemical parameters of studied molecules in aqueous phase are presented in Table 10. It is evident from Table 10 that BPP has the highest $E_{\mathrm{HOMO}}$ and a lower
$E_{\mathrm{HOMO}}$ in the protonated form. This means that the electron-donating ability of BPP is weaker in the protonated form. This confirms the experimental results that interaction between BPP and copper is electrostatic in nature (physisorption). The energy of the LUMO is directly related to the electron affinity and characterizes the susceptibility of the molecule towards attack by nucleophiles. The lower the values of $E_{\mathrm{LUMO}}$, the stronger the electron accepting abilities of molecules. It is clear that the protonated form of BPP exhibits the lowest $E_{\mathrm{HOMO}}$ thus, the protonated form is the most likely form for the interaction 

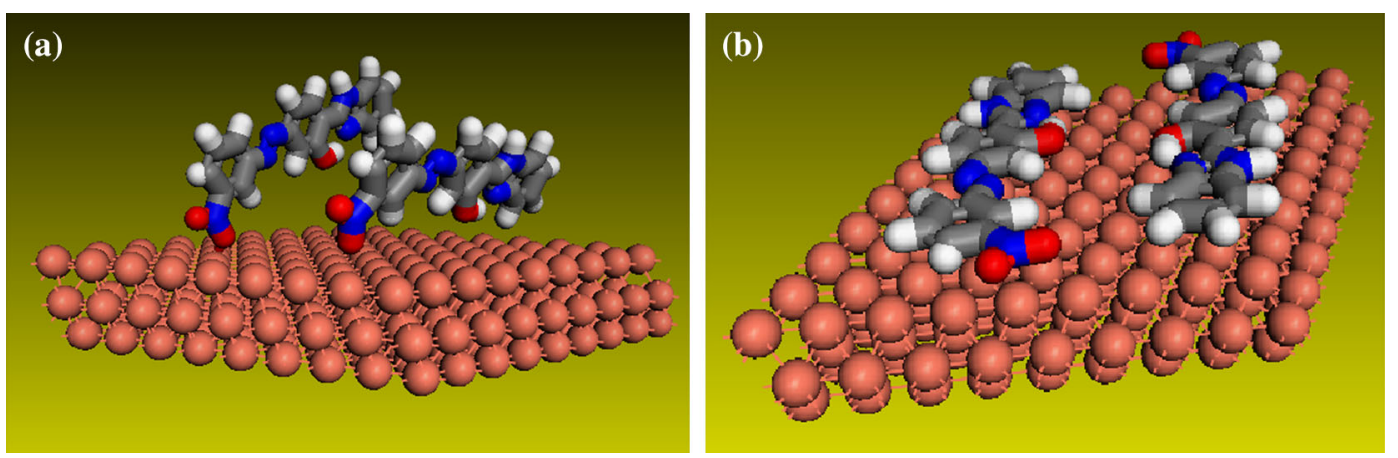

Fig. 17 The schematic proposed representation structure models of the adsorption behavior of the single BPP_1 inhibitor molecule on Cu (111) with different orientations in $2.0 \mathrm{M} \mathrm{HNO}_{3}$ solution

of copper with BPP molecule. Low values of the energy gap $(\Delta E)$ will provide good inhibition efficiencies, because the excitation energy to remove an electron from the last occupied orbital will be low [54]. Accordingly, the difference between $E_{\mathrm{LUMO}}$ and $E_{\mathrm{HOMO}}$ energy levels $\left(\Delta E=E_{\mathrm{LUMO}}-E_{\mathrm{HOMO}}\right)$ and the dipole moment $(\mu)$ was also determined. The global hardness $(\eta)$ is approximated as $\Delta E / 2$, and can be defined under the principle of chemical hardness and softness [14]. These parameters also provide information about the reactive behavior of molecules and are presented in Table 10. A molecule with a low-energy gap is more polarizable and is generally associated with a high chemical reactivity and low kinetic stability, and is termed soft molecule $[14,30]$. According to Wang et al. [46], adsorption of inhibitor onto a metallic surface occurs at the part of the molecule which has the greatest softness and lowest hardness. The results show that BPP in the protonated form have the lowest energy gap and lowest hardness; this agrees with the experimental results that BPP could have better inhibitive performance on copper surface, i.e., through electrostatic interaction between the cation form of BPP and the vacant $d$ orbital of copper physisorption. BPP had the highest inhibition efficiency because it had the highest HOMO energy values, and it had the greatest ability of offering electrons. This also agrees well with the value of $\left(\Delta G_{\text {ads }}^{\circ}\right)$ obtained experimentally (Table 7). The dipole moment of (BPP) is highest in the protonated form $[(\mu=9.5177 \quad$ Debye $\left.\left(28.818 \times 10^{-30} \mathrm{~cm}\right)\right]$, which is higher than that of $\mathrm{H}_{2} \mathrm{O}$ $\left(\mu=6.23 \times 10^{-30} \mathrm{~cm}\right)$. The high value of dipole moment probably increases the adsorption between chemical compound and metal surface [17, 19,30]. Accordingly, the adsorption of BPP molecules can be regarded as a quasisubstitution process between the benzimidazole compound and water molecules at the electrode surface. Frontier orbital energy level indicates the tendency of bonding to the metal surface. Further study on the formation of chelating centers in an inhibitor requires the information of spatial distribution of electronic density of the compound molecules [30]. The structure of the molecules can affect the adsorption by influencing the electron density at the functional group. Generally, electrophiles attack the molecules at negative charged sites. As seen from Fig. 16, the electron density focused on $\mathrm{N}$ atoms, $\mathrm{O}$ atoms, and $\mathrm{C}$ atoms in methyl. The regions of highest electron density are generally the sites to which electrophiles attacked. So, $\mathrm{N}, \mathrm{O}$, and $\mathrm{C}$ atoms were the active center, which had the strongest ability of bonding to the metal surface. On the other side, HOMO (Fig. 15) was mainly distributed on the areas containing imidazole; azo nitrogen. Thus, the areas containing $\mathrm{N}$ atoms were probably the primary sites of the bonding. As showed in Table 10, the values of HOMO energy increases with increasing length of carbon bone chain containing imidazole nitrogen. Similar situation can be also seen in Figs. 15 and 16 the configuration changes led to the increase in electron density; and inhibition efficiency was enhanced by increase in HOMO energy and electron density. The region of active centers transforming electrons from $\mathrm{N}$ atoms to copper surface. The electron configuration of copper is $[\mathrm{Ar}] 4 s^{2} 3 d^{9}$, the $3 d$ orbitals are not fully filled with electrons. $\mathrm{N}$ heteroatom's has lonely electron pairs that is important for bonding unfilled $3 d$ orbitals of $\mathrm{Cu}$ atom and determining the adsorption of the molecules on the metal surface. BPP_1 had the highest inhibition efficiency among the BPP_1-7, which was resulted from the geometry change that led to HOMO energy increase and electron density distribution in the molecule. Based on the discussion above, it can be concluded that the benzimidazole (BPP) molecules have many active centers of negative charge. In addition, the areas containing $\mathrm{N}$ and $\mathrm{O}$ atoms are the most possible sites of bonding metal surface by donating electrons to the copper surface.

According to HOMO and LUMO orbital energies given in Table 10, we can write the corrosion inhibition efficiency order as: $\quad$ BPP_1 $>$ BPP_2 $>$ BPP_3 $>$ 
BPP_4 $>$ BPP_5 $>$ BPP_6 $>$ BPP_7 (in terms of HOMO and LUMO energies). Chemical hardness is the resistance against electron cloud polarization or deformation of chemical species. As can be understood from this definition, chemical hardness of a molecule and its inhibition efficiency are inversely proportional to each other because a hard molecule is reluctant to give electrons. Chemical hardness, softness and $\Delta \mathrm{E}$ are quantum chemical parameters closely associated with each other [77]. As is known, both softness [78] and hardness are given based on HOMO and LUMO orbital energies as a result of Koopmans's theorem [18]. Hard molecules which have high HOMOLUMO energy gap cannot act as good corrosion inhibitor. However, soft molecules which have low HOMO-LUMO energy gap are good corrosion inhibitors because they can easily give to metals. It is clear that we can write the same corrosion inhibition ranking considering these three chemical properties. From the light of the results given in the Table 10, one can write the corrosion inhibition ranking of studied molecules based on their hardness, softness and HOMO-LUMO energy gap values as: BPP_1 $>$ BPP_2 $>$ BPP_3 $>$ BPP_4 $>$ BPP_5 $>$ BPP_6 $>$ BPP_7.

Electronegativity is an important parameter in terms of the prediction and comparison of corrosion inhibition efficiencies of molecules. The number of electrons transferred between metal and corrosion inhibitor can be calculated using Eq. (36). It is seen from the equation given below that the electron transfer value metal and inhibitor decreases as the electronegativity of inhibitor increases. According to Sanderson's electronegativity equalization principle [79], the electron transfer between metal and inhibitor continues until their electronegativity values become equal with each other. As a matter of fact, Eq. (36) has been derived taking advantage from hardness equalization principle and electronegativity equalization principle.

$\Delta N=\frac{\chi_{\mathrm{Cu}}-\chi_{\mathrm{inh}}}{2\left(\eta_{\mathrm{Cu}}+\eta_{\mathrm{inh}}\right)}$

where $\Delta N$ is electron transfer between metal and inhibitor. $X_{\mathrm{Cu}}$ and $\chi_{\text {inh }}$ are electronegativity of metal and electronegativity of inhibitor, respectively. $\eta_{\mathrm{Cu}}$ and $\eta_{\text {inh }}$ represent chemical hardness value of metal and chemical hardness value of inhibitor, respectively. Two systems, copper and inhibitor, are brought together, electrons will flow from lower $\chi$ (inhibitor) to higher $\chi(\mathrm{Cu})$, until the chemical potentials become equal. Copper surface is the Lewis acid according to HSAB theory [80]. The difference in electronegativity drives the electron transfer, and the sum of the hardness parameters acts as a resistance [81]. To calculate the fraction of electrons transferred, a theoretical value for the absolute electronegativity of copper according to Pearson was used $\chi_{\mathrm{Cu}}=463.1 \mathrm{~kJ} \mathrm{~mol}^{-1}$ [82], and a

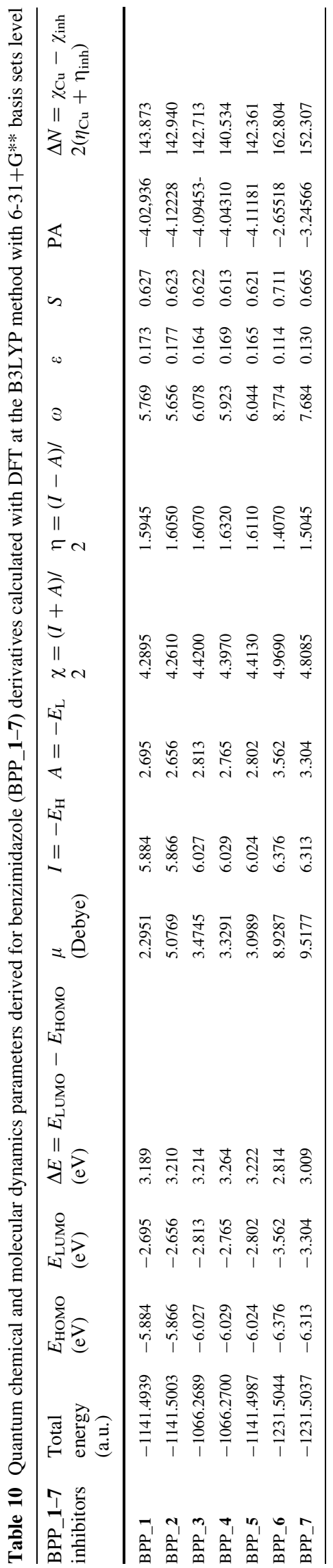


global hardness of $\eta_{\mathrm{Cu}}=0$, by assuming that for a metallic bulk $I=A$ [81] because they are softer than the neutral metallic atoms. From Table 10, it is possible to observe that molecule BPP_6 has a lower value of global hardness. The fraction of transferred electrons $(\Delta N)$ is also the largest for molecule BPP_6 then BPP_7 and, in turn, is $\mathrm{BPP} \_1, \approx \mathrm{BPP} \_2 \approx \mathrm{BPP} \mathbf{3} \approx \mathrm{BPP} \_5$, then $\mathrm{BPP} \_4$. Quantum chemical and molecular dynamics parameters derived for BPP_1-7 derivatives calculated with DFT at the B3LYP method with $6-31+\mathrm{G}^{* *}$ basis sets level are listed in Table 10. The values of the interaction energy and the total energy of the BPP_1-7 derivatives on copper (1 1 1) surface are listed in Table 10. It is clear from Table 10 that the total energy has a negative value, whereas the binding energy has a positive value (Table 7). As the value of the binding energy increases, the more easily the inhibitor adsorbs on the metal surface, the higher the inhibition efficiency [83]. BPP_1 has the highest binding energy compared to the other BPP derivatives to the copper surface that are found during the molecular dynamics simulation process described elsewhere [83]. High values of binding energy obtained with BPP_1 molecules explain its highest inhibition efficiency from the theoretical point of view. Therefore, according to a series of properties calculated for each molecule shown in Table 10 the reactivity order, that is, the inhibitive effectiveness order for the BPP molecules are: $-\mathrm{OCH}_{3}>-\mathrm{CH}_{3}>-\mathrm{NO}_{2}$ substituents. The calculated theoretical results are in agreement with experimental results. The use of Mulliken population analysis for the prediction of the adsorption center of inhibitors is widely used. The partial atomic charges on atoms of the inhibitor molecules provide important clues about the identifying of reactive center. The atoms with the highest negative charge represent the high tendency on the metal surface [78]. The inhibitors can easily interact with the metal surface through such atoms. Proton affinity can be defined as the enthalpy of the reaction with $\mathrm{H}^{+}$ion of a chemical species in gas phase and this parameter for inhibitors is one of the useful tools to compare their electron donating abilities [84]. The presence of the heteroatoms such as oxygen and nitrogen in the molecules of azole BPP_ 1-7 derivatives leads to high tendency for protonation in acidic medium. Thus, analysis of the protonated forms of BPP_1-7 derivatives is important in terms of the calculation of the proton affinities of neutral inhibitors. It should be stated that proton affinity is a measure of the basicity. In this sense, corrosion inhibitors act as Lewis bases. The basicity of a molecule will increase with increasing of its proton affinity. We calculated the proton affinities of the studied benzimidazole compounds considering Eqs. (37) and (38). According to proton affinity values given in the Table 10 for studied compounds, the inhibition efficiencies of mentioned compounds follow the same previous order.

$\mathrm{PA}=E_{(\text {pro })}-\left(E_{(\text {non-pro })}+E_{\mathrm{H}+}\right)$

where $E_{\text {non-pro }}$ and $E_{\text {pro }}$ are the energies of the non-protonated and protonated inhibitors, respectively. $E_{\mathrm{H}^{+}}$is the energy of $\mathrm{H}^{+}$ion and was calculated as:

$E_{\mathrm{H}^{+}}=E_{\left(\mathrm{H}_{3} \mathrm{O}^{+}\right)}-E_{\left(\mathrm{H}_{2} \mathrm{O}\right)}$

The electrophilicity index $(\omega)$ is an important parameter that indicates the tendency of the inhibitor molecule to accept the electrons. Nucleophilicity $(\varepsilon)$ is physically the inverse of electrophilicity $(1 / \omega)$. For this reason, it should be stated that a molecule that have large electrophilicity value is ineffective against corrosion while a molecule that have large nucleophilicity value is a good corrosion inhibitor. Thus, for studied molecules, we can write the inhibition efficiency ranking as: BPP_1 $>$ BPP_2 $>$ BPP_3 $>$ BPP_4 $>$ BPP_5 $>$ BPP_6 $>$ BPP_7.

\section{Molecular dynamics (MD) simulation}

The use of the molecular dynamics simulation is a useful and modern tool to investigate the interaction between inhibitors and metal surface. Thus, in this study, molecular dynamics simulation studies were performed to predict the binding energies of these azole derivatives on copper surface and to show whether there is a remarkable correlation between experimental inhibition efficiencies and binding energies for molecules considered in this study. The binding energies between $\mathrm{Cu}$ (111) surface and the benzimidazole (BPP) derivatives were obtained using Eq. (15). The schematic proposed representation structure models of the adsorption behavior of the single BPP_1 inhibitor molecule on $\mathrm{Cu}$ (111) substrate with different orientations in $2.0 \mathrm{M} \mathrm{HNO}_{3}$ solution has been presented in Fig. 17, i.e. representative snapshots of $\mathrm{BPP} \_1$ on $\mathrm{Cu}$ (111) surface (inset images show the on-top views). This indicates that the adsorption density of BPP_1 is higher and equilibrium adsorption configurations of the studied synthesized benzimidazole derivatives on $\mathrm{Cu}$ (111). Thus, Fig. 17 indicates the close contacts between the benzimidazole derivatives and $\mathrm{Cu}$ (111) metal surface as well as the best equilibrium adsorption configuration for the compounds considered. The obtained results given in Tables 7 and 10 show that the binding energies calculated for the interactions between inhibitors and metal surface are very high. It is important to note that high binding energy leads to a more stable inhibitor/surface interaction [85]. The binding energies obtained are observed to increase in the order: BPP_1 $>$ BPP_2 $>$ BPP_3 $>$ BPP_4 $>$ BPP_5 $>$ BPP_6 $>$ BPP_7. It is understood from the results, the benzimidazole 
(BPP_1-7) derivatives can act as good corrosion inhibitors against copper corrosion. It can also be seen from Fig. 11 and Table 7, there is a good linear correlation between experimental inhibition efficiencies and calculated binding energies in this study. Therefore, the studied BPP_1-7 molecules are likely to adsorb on the copper surface to form stable adsorbed layers and protect copper from corrosion.

The results obtained in the study showed that these compounds considered are good inhibitors against corrosion of copper. Quantum chemical calculations and molecular dynamics simulations carried out and the calculated binding energies of the studied molecules on copper surface demonstrated that these molecules are very effective against the corrosion of copper.

\section{Inhibition mechanism}

In acid solutions, organic inhibitors may interact with the corroding metal and hence affect the corrosion reaction in more than one way [86], sometimes simultaneously. It is, therefore, often difficult to assign a single general inhibition mechanism, since the mechanism may change with experimental conditions.

Both molecular and protonated species can adsorb on the copper surface. The adsorption of BPP derivatives through the lone pairs in the groups $\left(-\mathrm{N}=\mathrm{N}-,-\mathrm{OCH}_{3}\right.$, $\left.-\mathrm{OH},-\mathrm{NO}_{2},-\mathrm{NH}\right)$ can occur on the positive copper surface. Adsorption of the protonated BPP derivatives on the cathodic sites on copper surface will retard the oxygen evolution reaction. Adsorption on the anodic sites of copper surface can occur via $\mathrm{N}$ and $\mathrm{O}$ atoms to retard copper dissolution process. Adsorption of BPP derivatives on copper surface is assisted by hydrogen bond formation between BPP derivatives and the $\mathrm{Cu}_{2} \mathrm{O}$ and/or $\mathrm{CuO}$ formed on the copper surface. This type of adsorption should be more prevalent for protonated inhibitors, because the positive charge on the $\mathrm{N}$-atom is conductive to the formation of hydrogen bonds. Unprotonated $\mathrm{N}$ atoms may adsorb by direct chemisorption or by hydrogen bonding [87]. BPP derivatives, thus, have the ability to influence both the cathodic and anodic partial reactions, giving rise to the mixed-inhibition mechanism observed.

The well-known Pourbaix diagram (Fig. 8) for copperwater system, indicates that copper is corroded to $\mathrm{Cu}^{2+}$ in $\mathrm{HNO}_{3}$ solutions, and no oxide film is formed to protect the surface from corrosion. Copper dissolution is thus expected to be the dominant reaction in $\mathrm{HNO}_{3}$ solutions. The pure nitric acid and inhibitor-containing nitric acid solutions used in our experiments were all aerated where dissolved oxygen may be reduced on copper surface and this will allow some copper corrosion to occur [88]. It is a good approximation to ignore the hydrogen evolution reaction and only consider oxygen reduction in the aerated nitric acid solutions at potentials near the corrosion potential. Cathodic reduction of oxygen can be expressed either by a direct $4 \mathrm{e}^{-}$transfer, Eq. (25). Or by two consecutive $2 \mathrm{e}^{-}$ steps involving a reduction to hydrogen peroxide first, Eq. (39), followed by a further reduction, according to Eq. (40):

$\mathrm{O}_{2}+2 \mathrm{H}^{+}+2 \mathrm{e}^{-} \rightarrow \mathrm{H}_{2} \mathrm{O}_{2}$

$\mathrm{H}_{2} \mathrm{O}_{2}+2 \mathrm{H}^{+}+2 \mathrm{e}^{-} \rightarrow 2 \mathrm{H}_{2} \mathrm{O}$

The transfer of oxygen from the bulk solution to the copper/solution interface will strongly affect the rate of oxygen reduction reaction, despite how oxygen reduction takes place, either in $4 \mathrm{e}^{-}$transfer or two consecutive $2 \mathrm{e}^{-}$ transfer steps. Dissolution of copper in nitric acid is described by the following two continuous steps:

$\mathrm{Cu}-e^{-}=\mathrm{Cu}(\mathrm{I})_{\text {ads }} \quad$ (fast step)

$\mathrm{Cu}(\mathrm{I})_{\mathrm{ads}}-e^{-}=\mathrm{Cu}(\mathrm{II})_{\mathrm{ads}} \quad$ (slow step)

Where $\mathrm{Cu}(\mathrm{I})_{\mathrm{ads}}$ is an adsorbed species at the copper surface and does not diffuse into the bulk solution. The dissolution of copper is controlled by the diffusion of soluble $\mathrm{Cu}$ (II) species from the outer Helmholtz plane to the bulk solution. Upon addition of BPP, it is obvious that the slopes of the anodic $\left(\beta_{\mathrm{a}}\right)$ and cathodic $\left(\beta_{\mathrm{c}}\right)$ Tafel lines remain almost unchanged, giving rise to a nearly parallel set of anodic lines, and an almost parallel cathodic plots results too. Thus, the adsorbed BPP inhibitors act by simple blocking of the active sites for both anodic and cathodic processes. In other words, the adsorbed inhibitor decreases the surface area for corrosion without affecting the corrosion mechanism of copper in these solutions, and only causes inactivation of a part of the surface with respect to the corrosive medium [89]. From the experimental results obtained, we note that a plausible mechanism of corrosion inhibition of copper in $2.0 \mathrm{M} \mathrm{HNO}_{3}$ by BPP may be deduced on the basis of adsorption. In acidic solutions, the inhibitor can exist as cationic spices' (Eq. (43)) which may be adsorbed on the cathodic sites of copper and reduce the evolution of hydrogen:

$\mathrm{BPP}+2 \mathrm{H}^{+} \rightleftarrows[\mathrm{BPPH}]^{2+}$

The protonated BPP, however, could be attached to the copper surface by means of electrostatic interaction between $\mathrm{NO}_{3}{ }^{-}$and protonated BPP since the copper surface has positive charges in the acid medium [90]. This could further be explained based on the assumption that in the presence of $\mathrm{NO}_{3}^{-}$, the negatively charged $\mathrm{NO}_{3}{ }^{-}$would attach to positively charged surface. When BPP molecule adsorbs on the copper surface, electrostatic interaction takes place by partial transference of electrons from the 
polar atoms $(\mathrm{N}$ and $\mathrm{O}$ atoms and the delocalized $\pi$-electrons around the heterocyclic ring) of azole molecules to the metal surface. In addition to electrostatic interaction (physisorption) of BPP molecules on the copper surface, molecular adsorption may also play a role in the adsorption process. The inhibition of copper corrosion in acid solution by the investigated 2-(2-benzimidazolyl)-4(phenylazo) phenol derivatives was found to depend on the concentration and nature of the inhibitor. Variation in structure of the inhibitor molecules takes place through the arylazo side chain, the benzimidazolyl moiety being the same. The electron charge density of the adsorption centers would depend on substituents in the arylazo conjugated with the benzimidazolyl. Also, substituents on the phenyl ring may participate in adsorption. Depending on polar substituent constant $(\sigma)$, the electron-donating character of the substituents used is increased in the order: $\mathrm{OCH}_{3}>\mathrm{CH}_{3}>$ $\mathrm{NO}_{2}$. This order is almost concordant with the observed order of inhibition efficiency IE\% for our BPP compounds. The $\mathrm{o}-\mathrm{OCH}_{3}$ compound is the most efficient inhibitor. Owing to the higher electron donating character of $-\mathrm{OCH}_{3}$ compared to the other substituents. On the other hand, $\mathrm{p}-\mathrm{NO}_{2}$ and $\mathrm{m}-\mathrm{NO}_{2}$ compounds were the least effective inhibitors in this series. This may be attributed to the planarity of the $\mathrm{p}-\mathrm{NO}_{2}$ and $\mathrm{m}-\mathrm{NO}_{2}$ groups with the phenyl ring brings about maximum electron withdrawal. Also, the $\mathrm{NO}_{2}$ group is easily reduced in acid medium and this process is exothermic, heat evolved aids desorption of the compound on copper surface. o- $\mathrm{CH} 3$ and $\mathrm{m}-\mathrm{CH}_{3}$ compounds comes after $\mathrm{p}-\mathrm{OCH}_{3}$ and $\mathrm{o}-\mathrm{OCH}_{3}$ compound in inhibition efficiency. This is due to the lower electron-donating character of $-\mathrm{CH}_{3}$ group compared to $-\mathrm{OCH}_{3}$ group. Thus, the overall mechanism constituents electron release which maintains sufficient electron charge density on the molecule.

\section{Conclusions}

The following conclusions may be drawn from the study:

1. 2-(2-benzimidazolyl)-4 (phenylazo) phenol (BPP_1-7) derivatives were found to act as safe effective corrosion inhibitors for copper surface in $2.0 \mathrm{M} \mathrm{HNO}_{3}$. Inhibition efficiency (IE\%) values increase with the inhibitor concentration but decrease with rise in temperature suggesting physical adsorption, with some chemisorption $\left(\Delta G_{\text {ads }}^{\circ}\right.$ more negative than $-40 \mathrm{~kJ} / \mathrm{mol}$ ). Moreover, the inhibition of copper by BPP_1-7 substituents is often explained by the formation of $\mathrm{Cu}$ (II)-BPP through its heteroatoms.
2. Polarization measurements show that BPP_1-7 derivatives act essentially as a mixed-type inhibitors and their inhibition mechanism is adsorption assisted by hydrogen bond formation.

3. The adsorption of BPP_1-7 inhibitors on copper surface was found to accord with Frumkin adsorption isotherm model. The adsorption process is strongly, spontaneously, exothermic and accompanied with an increase in entropy of the system from thermodynamic point of view.

4. Phenomenon of adsorption is proposed from the values of kinetic/thermodynamics parameters $\left(\Delta G_{\mathrm{ads}}^{\circ}, 1 / y, K_{\mathrm{b}}\right.$, $f, K_{\mathrm{ads}}, E_{\mathrm{a}}, \Delta H^{*}, \Delta S^{*}$ and $\left.\Delta G^{*}\right)$ obtained.

5. The theoretical quantum study demonstrated that the inhibition efficiency increases with increase in $E_{\mathrm{HOMO}}$ and decrease in $E_{\mathrm{LUMO}}$, dipole moment $(\mu)$ and energy gap $\Delta E$. The quantum mechanical approach may well be able to foretell molecule structures that are better for corrosion inhibition.

6. Equilibrium adsorption configurations of the studied synthesized BPP_1 derivative on $\mathrm{Cu}$ (111) has been presented.

7. The efficiency order of the studied benzimidazole BPP_1-7 inhibitors obtained by experimental results was verified by theoretical calculations.

Acknowledgments I gratefully acknowledge Tanta University, Chemistry Department, at Tanta, Egypt for the financial assistance and Department of Chemistry, Al-Baha University, Baljarashi, Saudi Arabia for facilitation of our study.

Open Access This article is distributed under the terms of the Creative Commons Attribution 4.0 International License (http://crea tivecommons.org/licenses/by/4.0/), which permits unrestricted use, distribution, and reproduction in any medium, provided you give appropriate credit to the original author(s) and the source, provide a link to the Creative Commons license, and indicate if changes were made.

\section{References}

1. Krishnamoorthy A, Chanda K, Murarka SP, Ramanath G, Ryan JG (2001) Self-assembled near-zero-thickness molecular layers as diffusion barriers for $\mathrm{Cu}$ metallization. Appl Phys Lett $78: 2467-2469$

2. Khaled KF (2010) Corrosion control of copper in nitric acid solutions using some amino acids: a combined experimental and theoretical study. Corros Sci 52:3225-3234

3. Mihit M, Laarej K, Abou El Makarim H, Bazzi L, Salghi R, Hammouti B (2010) Study of the inhibition of the corrosion of copper and zinc in $\mathrm{HNO}_{3}$ solution by electrochemical technique and quantum chemical calculations. Arab J Chem 3:55-60

4. Parr RG, Yang W (1989) Density functional theory of atoms and molecules. Oxford University Press, Oxford UK 
5. Dreizler RM, Gross EKU (1990) Density functional theory. Springer, Berlin

6. Obot IB, Ebenso EE, Obi-Egbedi NO, Afolabi AS, Gasem ZM (2012) Experimental and theoretical investigations of adsorption characteristics of itraconazole as green corrosion inhibitor at a mild steel/hydrochloric acid interface. Res Chem Intermed 38:1761-1779

7. Khaled KF (2008) Molecular simulation, quantum chemical calculations and electrochemical studies for inhibition of mild steel by triazoles. Electrochim Acta 53:3484-3492

8. Obot IB, Ebenso EE, Kabanda MM (2013) Metronidazole as environmentally safe corrosion inhibitor for mild steel in $0.5 \mathrm{M}$ $\mathrm{HCl}$ : experimental and theoretical investigation. J Environ Chem Eng 1:431-439

9. Kabanda MM, Obot IB, Ebenso EE (2013) Computational study of some amino acid derivatives as potential corrosion inhibitors for different metal surfaces and in different media. Int $\mathbf{J}$ Electrochem Sci 8:10839-10850

10. Obot IB, Obi-Egbedi NO (2010) Theoretical study of benzimidazole and its derivatives and their potential activity as corrosion inhibitors. Corros Sci 52:657-660

11. Obi-Egbedi NO, Obot IB, El-Khaiary MI, Umoren SA, Ebenso EE (2012) Computational simulation and statistical analysis on the relationship between corrosion inhibition efficiency and molecular structure of some phenanthroline derivatives on mild steel surface. Int J Electrochem Sci 7:5649-5675

12. Kaya S, Kaya C (2015) A new equation for calculation of chemical hardness of groups and molecules. Mol Phys 113:1311-1319

13. Kaya S, Kaya C (1060) A new method for calculation of molecular hardness: a theoretical study. Comput Theor Chem 2015:66-70

14. Yang W, Parr RG (1985) Hardness, softness and the Fukui function in the electronic theory of metals and catalysis. Proc Natl Acad Sci 82:6723-6726

15. Kaya S, Kaya C (1052) A new equation based on ionization energies and electron affinities of atoms for calculating of group electronegativity. Comput Theor Chem 2015:42-46

16. Safi ZS, Omar S (2014) Proton affinity and molecular basicity of $m$ - and $p$-substituted benzamides in gas phase and in solution: a theoretical study. Chem Phys Lett 610-611:321-330

17. Chattaraj PK, Sarkar U, Roy DR (2006) Electrophilicity Index. Chem. Rev. 106(6):2065-2091

18. Koopmans T (1933) Ordering of wave functions and eigen-energies to the individual electrons of an atom. Physica 1:104-113

19. Chattaraj PK, Lee H, Parr RG, Principle HSAB (1991) J Am Chem Soc 113(5):1855-1856

20. Pearson RG (1997) Chemical hardness: applications from molecules to solids. Wiley-VCH, Weinheim

21. Zhang F, Tang Y, Cao Z, Jing W, Wu Z, Chen Y (2012) Performance and theoretical study on corrosion inhibition of 2-(4pyridyl)-benzimidazole for mild steel in hydrochloric acid. Corros Sci 61:1-9

22. Jiang L, Lan Y, He Y, Li Y, Li Y, Luo J (2014) 1,2,4-Triazole as a corrosion inhibitor in copper chemical mechanical polishing. Thin Solid Films 556:395-404

23. Ramezanzadeh B, Arman SY, Mehdipour M, Markhali BP (2014) Analysis of electrochemical noise (ECN) data in time and frequency domain for comparison corrosion inhibition of some azole compounds on $\mathrm{Cu}$ in $1.0 \mathrm{M} \mathrm{H}_{2} \mathrm{SO}_{4}$ solution. Appl Surf Sci 289:129-140

24. Kuznetsov YI, Agafonkina MO, Andreeva NP (2014) Mercaptobenzimidazole (MBIMD) physical and chemical adsorption with $\Delta \mathrm{G}$ of $-31.1 \mathrm{~kJ}$ mol. Russ J Phys Chem $\mathrm{A}+88: 702$
25. Liu S, Duan JM, Jiang RY, Feng ZP, Xiao R (2011) Corrosion inhibition of copper in tetra- $n$-butylammonium bromide aqueous solution by benzotriazole. Mater Corros 62:47-52

26. Marija B, Mihajlović P, Antonijević MM (2015) Copper corrosion inhibitors period 2008-2014: a review. Int J Electrochem Sci 10:1027-1053

27. Antonijevic MM, Petrovic MB (2008) Copper corrosion inhibitors: a review. Int J Electrochem Sci 3:1-28

28. Ahamad I, Quraishi MA (2009) Bis (benzimidazol-2-yl) disulphide: an efficient water soluble inhibitor for corrosion of mild steel in acid media. Corros Sci 51(9):2006-2013

29. Ahamad I, Quraishi MA (2010) Mebendazole: new and efficient corrosion inhibitor for mild steel in acid medium. Corros Sci 52(2):651-656

30. Madkour LH, Elroby SK (2015) Inhibitive properties, thermodynamic, kinetics and quantum chemical calculations of polydentate Schiff base compounds as corrosion inhibitors for iron in acidic and alkaline media. Int J Indus Chem 6(3):165-184

31. Maayta AK, Al-Rawashded NAF (2004) Inhibition of acidic corrosion of pure aluminum by some organic compounds. Corros Sci 46(5):1129-1140

32. Emregül KC, Akay AA, Atakol O (2005) The corrosion inhibition of steel with Schiff base compounds in $2 \mathrm{M} \mathrm{HCl}$. Mater Chem Phys 93:325-329

33. Madkour LH, Elmorsi MA, Ghoneim MM (1995) Inhibition of copper corrosion by arylazotriazoles in nitric acid solution. Monatshefte fiir Chemie 126:1087-1095

34. Madkou LH, Ghoneim MM (1997) Inhibition of the corrosion of $16 / 14$ austenitic stainless steel by oxygen and nitrogen containing compounds. Bull Electrochem 13(1):1-7

35. Gece Gökhan (2008) The use of quantum chemical methods in corrosion inhibitor studies. Corros Sci 50(11):2981-2992

36. JiaJun Fu, Zang HaiShan, Wang Ying, Li SuNing, Chen Tao, Liu XiaoDong (2012) Experimental and theoretical study on the inhibition performances of quinoxaline and its derivatives for the corrosion of mild steel in hydrochloric acid. Ind Eng Chem Res 51(18):6377-6386

37. FuSu-ning Jia-jun, Li Su-Ning, Wang Ying, Liu Xiao-Dong, LuDe Lu (2011) Computational and electrochemical studies on the inhibition of corrosion of mild steel by 1 Cysteine and its derivatives. J Mater Sci 46(10):3550-3559

38. Frisch MJ, Trucks GW, Schlegel HB, Scuseria GE, Robb MA, Cheeseman JR, Scalmani G, Barone V, Mennucci B, Petersson GA, Nakatsuji H, Caricato M, Li X, Hratchian HP, Izmaylov AF, Bloino J, Zheng G, Sonnenberg JL, Hada M, Ehara M, Toyota K, Fukuda R, Hasegawa J, Ishida M, Nakajima T, Honda Y, Kitao O, Nakai H, Vreven T, Montgomery JA Jr, Peralta JE, Ogliaro F, Bearpark M, Heyd JJ, Brothers E, Kudin KN, Staroverov VN, Kobayashi R, Normand J, Raghavachari K, Rendell A, Burant JC, Iyengar SS, Tomasi J, Cossi M, Rega N, Millam JM, Klene M, Knox JE, Cross JB, Bakken V, Adamo C, Jaramillo J, Gomperts R, Stratmann RE, Yazyev O, Austin AJ, Cammi R, Pomelli C, Ochterski JW, Martin RL, Morokuma K, Zakrzewski VG, Voth GA, Salvador P, Dannenberg JJ, Dapprich S, Daniels AD, Farkas O, Foresman JB, Ortiz JV, Cioslowski J, Fox DJ, Inc Gaussian, Wallingford CT (2009) Gaussian 09. Gaussian Inc, Pittsburgh PA, p 2009

39. Lee C, Yang W, Parr RG (1988) Development of the ColleSalvetti correlation-energy formula into a functional of the electron density. Phys Rev B 37:785-789

40. Becke AD (1993) Density-functional thermochemistry. III. The role of exact exchange. J Chem Phys 98:5648-5652

41. Perdew JP, Burke K, Ernzerhof M (1997) Generalized gradient approximation made simple. Phys Rev Lett 78:1396 
42. Perdew JP, Burke K, Ernzerhof M (1996) Generalized gradient approximation made simple. Phys Rev Lett 77:3865-3868

43. Chermette H (1999) Chemical reactivity indexes in density functional theory. J Comput Chem 20:129-154

44. Parr RG, Chattaraj PK (1991) Principle of maximum hardness. J Am Chem Soc 113:1854-1855

45. Islam N, Ghosh DC (2011) A new algorithm for the evaluation of the global hardness of polyatomic molecules. Int J Quant Chem 109:917-931

46. Parr RG, Sventpaly L, Liu S (1999) Electrophilicity index. J Am Chem Soc 121(9):1922-1924

47. Kirkpatrick S, Gelatt CD, Vecchi MP (1983) Optimization by simulated annealing. Science 220(4598):671-680

48. Sun H (1998) COMPASS: an ab initio force-field optimized for condensed-phase applications: overview with details on alkane and benzene compounds. J Phys Chem B 102(38):7338-7364

49. Guoa Lei, Zhub Shanhong, Zhang Shengtao (2015) Experimental and theoretical studies of benzalkonium chloride as an inhibitor for carbon steel corrosion in sulfuric acid. J Ind Eng Chem 24:174-180

50. Zhou Y, Xu S, Guo L, Zhang S, Lu H, Gong Y, Gao G (2015) Evaluating two new Schiff bases synthesized on the inhibition of corrosion of copper in $\mathrm{NaCl}$ solutions. RSC Adv 5:14804-14813

51. Strehblow HH, Titze B (1980) The investigation of the passive behaviour of copper in weakly acid and alkaline solutions and the examination of the passive film by Esca and ISS. Electrochim Acta 25(6):839-850

52. Brusic V, Frisch MA, Eldridge BN, Novak FP, Kaufman FB, Rush BM, Frankel GS (1991) Copper corrosion with and without inhibitors. J Electrochem Soc 138:2253-2259

53. Pourbaix M (1975) Atlas of electrochemical equilibria in aqueous solutions. NACE, Houston

54. Khaled KF, Fadl-Allah SA, Hammouti B (2009) Some benzotriazole derivatives as corrosion inhibitors for copper in acidic medium: experimental and quantum chemical molecular dynamics approach. Mater Chem Phys 117(1):148-155

55. McCafferty E (2005) Validation of corrosion rates measured by the Tafel extrapolation method. Corros Sci 47(12):3202-3215

56. Quartarone G, Battilana M, Bonaldo L, Tortato T (2008) Investigation of the inhibition effect of indole-3-carboxylic acid on the copper corrosion in $0.5 \mathrm{M} \mathrm{H}_{2} \mathrm{SO}_{4}$. Corros Sci 50(12):3467-3474

57. Amin MA, Khaled KF (2010) Copper corrosion inhibition in $\mathrm{O}_{2}$ saturated $\mathrm{H}_{2} \mathrm{SO}_{4}$ solutions. Corros Sci 52(4):1194-1204

58. Quartarone G, Bellomi T, Zingales A (2003) Inhibition of copper corrosion by isatin in aerated $0.5 \mathrm{M} \mathrm{H}_{2} \mathrm{SO}_{4}$. Corros Sci 45(4):715-733

59. Behpour M, Ghoreishi SM, Soltani N, Salavati-Niasari M, Hamadanian M, Gandomi A (2008) Electrochemical and theoretical investigation on the corrosion inhibition of mild steel by thiosalicylaldehyde derivatives in hydrochloric acid solution. Corros Sci 50(8):2172-2181

60. Musa AY, Kadhum AAH, Mohamad AB, Daud AR, Takriff MS, Kamarudin SK (2009) A comparative study of the corrosion inhibition of mild steel in sulphuric acid by4,4-dimethyloxazolidine-2-thione. Corros Sci 51(10):2393-2399

61. Ye XR, Xin XQ, Zhu JJ, Xue ZL (1998) Coordination compound films of 1-phenyl-5-mercaptotetrazole on copper surface. Appl Surf Sci 135:307-317

62. Benali O, Larabi L, Traisnel M, Gengembra L, Harek Y (2007) Electrochemical, theoretical and XPS studies of 2-mercapto-1methylimidazole adsorption on carbon steel in $1 \mathrm{M} \mathrm{HClO}_{4}$. Appl Surf Sci 253(14):6130-6139

63. Noor EA, Al-Moubaraki AH (2008) Thermodynamic study of metal corrosion and inhibitor adsorption processes in mild steel/ 1-methyl-4[4' $(-X)$-styryl pyridinium iodides/hydrochloric acid systems. Mater Chem Phys 110(1):145-154
64. Yadav DK, Maiti B, Quraishi MA (2010) Electrochemical and quantum chemical studies of 3,4-dihydropyrimidin-2(1H)-ones as corrosion inhibitors for mild steel in hydrochloric acid solution. Corros Sci 52(11):3586-3598

65. Badawy WA, Ismail KM, Fathi AM (2006) Corrosion control of $\mathrm{Cu}-\mathrm{Ni}$ alloys in neutral chloride solutions by amino acids. Electrochim Acta 51(20):4182-4189

66. Tang L, Li X, Si Y, Mu G, Liu G (2006) The synergistic inhibition between 8-hydroxyquinoline and chloride ion for the corrosion of cold rolled steel in $0.5 \mathrm{M}$ sulfuric acid. Mater Chem Phys 95(1):29-38

67. Abdallah M (2002) Rhodanine azosulpha drugs as corrosion inhibitors for corrosion of 304 stainless steel in hydrochloric acid solution. Corros Sci 44(4):717-728

68. El-Awady AA, Abd-El-Nabey BA, Aziz SG (1992) Kineticthermodynamic and adsorption isotherms analyses for the inhibition of the acid corrosion of steel by cyclic and open-chain amines. J Electrochem Soc 139(8):2149-2154

69. Popova A, Sokolova E, Raicheva S, Christov M (2003) AC and DC study of the temperature effect on mild steel corrosion in acid media in the presence of benzimidazole derivatives. Corros Sci 45(1):33-58

70. Shukla SK, Quraishi MA (2009) Cefotaxime sodium: a new and efficient corrosion inhibitor for mild steel in hydrochloric acid solution. Corros Sci 51(5):1007-1011

71. Singh AK, Quraishi MA (2010) Effect of Cefazolin on the corrosion of mild steel in $\mathrm{HCl}$ solution. Corros Sci 52(1):152-160

72. Umoren SA, Ekanem UF (2010) Inhibition of mild steel corrosion in $\mathrm{H} 2 \mathrm{SO} 4$ using exudate gum from Pachylobus edulis and synergistic potassium halide additives. Chem Eng Commun 197(10):1339-1356

73. Fouda AS, Al-Sarawy AA, El-Katori EE (2006) Pyrazolone derivatives as corrosion inhibitors for $\mathrm{C}$-steel in hydrochloric acid solution. Desalination 201(1-3):1-13

74. Guan NM, Xueming L, Fei L (2004) Mater Chem Phys 86:59-68

75. Li Xianghong, Deng Shuduan (2009) Hui Fu., Synergism between red tetrazolium and uracil on the corrosion of cold rolled steel in $\mathrm{H}_{2} \mathrm{SO}_{4}$ solution. Corros Sci 51(6):1344-1355

76. Taqui Khan MM, Shukla RS (1991) Kinetic and spectroscopic study of the formation of an intermediate ruthenium(III) ascorbate complex in the oxidation of L-ascorbic acid. Polyhedron 10(23-24):2711-2715

77. Obot IB, Obi-Egbedi NO, Eseola AO (2011) Anticorrosion potential of 2-mesityl-1H-imidazo[4,5-f][1,10]-phenanthroline on mild steel in sulfuric acid solution: experimental and theoretical study. Ind Eng Chem Res 50:2098-2110

78. Obi-Egbedi NO, Obot IB, El-Khaiary MI (1002) Quantum chemical investigation and statistical analysis of the relationship between corrosion inhibition efficiency and molecular structure of xanthene and its derivatives on mild steel in sulphuric acid. J Mol Struct 2011:86-96

79. Sanderson RT (1976) Chemical bond and bond energy. Academic Press, New York

80. Pearson RG (1988) Absolute electronegativity and hardness: application to inorganic chemistry. Inorg Chem 27(4):734-740

81. Rodríguez-Valdez LM, Martínez-Villafañe A, Glossman-Mitnik D (2005) CHIH-DFT theoretical study of isomeric thiatriazoles and their potential activity as corrosion inhibitors. J Mol Struct Thechem 716(1-3):61-65

82. Sastri VS, Perumareddi JR (1997) Molecular orbital theoretical studies of some organic corrosion inhibitors. Corros Sci 53(8):617-622

83. Khaled KF (2009) Monte Carlo simulations of corrosion inhibition of mild steel in $0.5 \mathrm{M}$ sulphuric acid by some green corrosion inhibitors. J Solid State Electrochem 13:1743-1756 
84. Kaya C (2011) Inorganic chemistry 1 and 2. Palme Publishing, Ankara

85. John S, Joy J, Prajila M, Joseph A (2011) Electrochemical, quantum chemical and molecular dynamics studies on the interaction of 4-amino-4H,3,5- di(methoxy)-1,2,4-triazole (ATD), BATD, and DBATD on copper metal in $1 \mathrm{~N} \mathrm{H}_{2} \mathrm{SO}_{4}$. Mater Corros 62:1031-1041

86. Oguzie EE, Onuoha GN, Onuchukwu AI (2005) Inhibitory mechanism of mild steel corrosion in $2 \mathrm{M}$ sulphuric acid solution by methylene blue dye. Mater Chem Phys 89:305-311

87. Khaled KF, Amin MA (2008) Computational and electrochemical investigation for corrosion inhibition of nickel in molar nitric acid by piperidines. J Appl Electrochem 38:1609-1621
88. Quartarone G, Moretti G, Bellomi T, Capobianco G, Zingales A (1998) Using indole to inhibit copper corrosion in aerated $0.5 \mathrm{M}$ sulfuric acid. Corrosion 54(8):606-618

89. Ashassi-Sorkhabi H, Ghalebsaz-Jeddi N, Hashemzadeh F, Jahani $\mathrm{H}$ (2006) Corrosion inhibition of carbon steel in hydrochloric acid by some polyethylene glycols. Electrochim Acta 51(18):3848-3854

90. Li Y, Zhao P, Liang Q, Hou B (2005) Berberine as a natural source inhibitor for mild steel in $1 \mathrm{M} \mathrm{H}_{2} \mathrm{SO}_{4}$. Appl Surf Sci 252(5):1245-1253 